\title{
PROCEDIMIENTO DE DISEÑO SISMICO MULTINIVEL BASADO EN DESPLAZAMIENTO PARA ESTRUCTURAS A BASE DE MARCOS DE CONCRETO REFORZADO
}

\author{
José E. Barradas Hernández ${ }^{(1)}$ y A. Gustavo Ayala Milián (1)
}

\begin{abstract}
RESUMEN
Se propone un procedimiento de diseño sísmico multinivel basado en desplazamientos, aplicable a estructuras conformadas por marcos de concreto reforzado. Mediante la aplicación de este procedimiento se satisfacen simultáneamente los requisitos de rigidez para el estado límite de servicio, de resistencia para un estado límite auxiliar, denominado de fluencia incipiente, y la capacidad de deformación inelástica para el estado límite de prevención del colapso. El procedimiento se fundamenta en conceptos básicos de la dinámica estructural y hace uso de análisis modales espectrales, por lo que se puede ejecutar con el uso de programas comerciales de análisis estructural, siendo de esta forma viable su implementación en la práctica del diseño. Para ilustrar y validar el procedimiento, se diseñan tres marcos de concreto reforzado y los índices de desempeño establecidos en el diseño se comparan con los obtenidos de un análisis inelástico paso a paso ante las acciones sísmicas de diseño. Se muestra que el procedimiento propuesto es capaz de predecir con suficiente precisión las máximas demandas de deformación lateral establecidas en el diseño.
\end{abstract}

Palabras clave: diseño multinivel; diseño símico basado en desplazamientos; modos superiores

\section{A DISPLACEMENT-BASED MULTI-LEVEL SEISMIC DESIGN METHOD FOR REINFORCED CONCRETE FRAME STRUCTURES}

\begin{abstract}
A multi-level displacement-based seismic design procedure applicable to reinforced concrete frame structures is presented. This procedure simultaneously satisfies the stiffness requirements for the serviceability limit state, of strength for an auxiliary limit state, called of incipient yielding, besides the inelastic deformation capacity for the collapse prevention limit state. The procedure is based on basic concepts of structural dynamics and uses modal spectral analyses, so that it can be executed with commercial structural analysis software used in routine structural design. It is shown that this procedure is capable of predicting the inelastic deformation demands in a simpler and more effective way than other displacement-based procedures. To illustrate and validate the procedure, three reinforced concrete frames structures are designed, and target performance indices are compared with those obtained from inelastic step by step analyses under the design seismic actions. It is shown that the proposed procedure yields maximum demands of inelastic lateral deformations reasonably close to those established for the design.
\end{abstract}

Keywords: multi-level design; displacement-based seismic design; higher mode effects

Artículo recibido el 5 de agosto de 2013 y aprobado para su publicación el 11 de junio de 2014. Se aceptarán comentarios y/o discusiones hasta cinco meses después de su publicación.

(1) Instituto de Ingeniería, Universidad Nacional Autónoma de México, Coyoacán, C.P. 04510, México, D.F. JBarradasH@@iingen.unam.mx GAyalaM@@iingen.unam.mx 


\section{INTRODUCCIÓN}

La filosofía de diseño sísmico adoptada en la mayoría de los reglamentos internacionales establece que un edificio y sus componentes no estructurales deben de satisfacer los siguientes criterios de desempeño: (1) presentar un bajo nivel de daño para preservar su funcionalidad ante sismos de baja intensidad de frecuente ocurrencia, y (2), no colapsar ante los sismos de mayor intensidad que puedan ocurrir para proteger la vida de los ocupantes. En algunas normas de diseño sísmico, como el Reglamento de Construcciones del Distrito Federal del 2004 (RCDF-2004), (GDF, 2004), al primer estado de daño se le denomina estado límite de servicio (ELS), y al segundo, estado límite de falla o de prevención del colapso (ELPC). El cumplimiento de ambos estados límite ante sus correspondientes sismos de diseño define el objetivo de desempeño (OD), y al criterio de satisfacer dos o más estados límite se le denomina diseño sísmico multinivel.

Eventos sísmicos de diferentes intensidades ocurridos en décadas recientes en varios países, han puesto en evidencia que la aplicación del método de análisis y diseño sísmico estipulado en los reglamentos, usualmente denominado "basado en fuerzas", no garantiza que se cumpla el OD. Esto se debe principalmente a que los valores estipulados por los reglamentos para la capacidad dúctil y sobrerresistencia de estructuras, son de naturaleza completamente empírica (Uang, 1991).Estos hechos han impulsado el desarrollo de procedimientos de análisis y diseño sísmico opcionales, denominados como "basados en desempeño", con los cuales se busca garantizar que se cumpla el OD. Actualmente existe una variedad de métodos de diseño sísmico basados en desempeño, comúnmente clasificados de acuerdo con los índices de desempeño para los que se diseña la estructura, y de entre los cuales han destacado los basados en desplazamientos. Esto se debe tanto a la transparencia y sencillez de su planteamiento, como a la relativa eficacia obtenida de su uso para controlar el daño en las edificaciones, ya que se ha observado que existe una buena correlación entre las distorsiones de entrepiso de una estructura, las deformaciones angulares y capacidad rotacional de sus elementos estructurales (Qi y Moehle, 1991; Moehle, 1992), y la magnitud del daño en sus elementos no estructurales (Mayes, 1995).

A pesar de la variedad de métodos basados en desempeño existentes en la actualidad, poco plantean un esquema de diseño sísmico multinivel en el que se considere el cumplimiento del ELS, o en general, un estado para el que la respuesta de la estructura se encuentre por debajo de su límite de comportamiento elástico. Más aún, algunos de estos procedimientos son conceptualmente complejos (Bertero, 2001), o se apoyan en el uso de análisis dinámicos no lineales paso a paso (Kappos, 2001), por lo que no es factible su implementación en la práctica del diseño.

Este artículo presenta un procedimiento de diseño sísmico multinivel basado en desplazamientos aplicable a estructuras conformadas por marcos de concreto reforzado, con el que se satisfacen simultáneamente los requerimientos de rigidez del ELS, de resistencia para un estado límite auxiliar asociado a la fluencia incipiente del sistema estructural (EFI),así como la capacidad de deformación inelástica requerida para el ELPC. El procedimiento se fundamenta en conceptos básicos de la dinámica estructural y se apoya en el uso de análisis modales espectrales, por lo que es conceptualmente sencillo y fácil de aplicar mediante programas comerciales de análisis estructural. Estas características lo hacen viable de implementar en la práctica del diseño sísmico. Para ilustrar y validar el procedimiento propuesto, se diseñan cuatro marcos de concreto reforzado y se comparan los índices de desempeño establecidos en el diseño, con los obtenidos de un análisis dinámico inelástico paso a paso de las estructuras diseñadas para las acciones sísmicas de diseño. 


\section{FUNDAMENTOS DEL MÉTODO PROPUESTO}

Un oscilador de un grado de libertad (1GDL) con comportamiento inelástico idealizado como bilineal que no presenta degradación de rigidez ni de resistencia, se caracteriza por su frecuencia elástica $\left(\omega_{E}\right)$, resistencia de fluencia por unidad de masa $(R y / m)$, y frecuencia de posfluencia $\left(\alpha \omega_{E}\right)$, donde $\alpha$, cuando el oscilador no presenta un significativo ablandamiento por efectos $\mathrm{P}-\Delta$, es la relación positiva de rigidez de posfluencia a rigidez inicial. Dadas estas propiedades, el oscilador desarrollará ante una acción sísmica un desplazamiento de fluencia $(S d y)$, una demanda máxima de ductilidad $(\mu)$, un desplazamiento inelástico máximo ( $S d m a x)$, y una correspondiente resistencia máxima por unidad de masa $(R m a x / m)$. Las propiedades y la respuesta ante fuerzas cíclicas reversibles de este oscilador se pueden representar en un espacio espectral pseudoaceleración $(S a)$ versus desplazamiento $(S d)$, como una curva bilineal, fig. 1, denominada en el presente estudio como curva de comportamiento (curva base) del oscilador de 1GDL.El idealizar el comportamiento del oscilador como bilineal, permite que su respuesta máxima $(R \max / m, S d m a x)$ se pueda aproximar como la suma de su respuesta elástica $(R y / m, S d y)$ y una respuesta adicional, denominada en este estudio como respuesta complementaria, (Rcomp/m, Sdcomp).

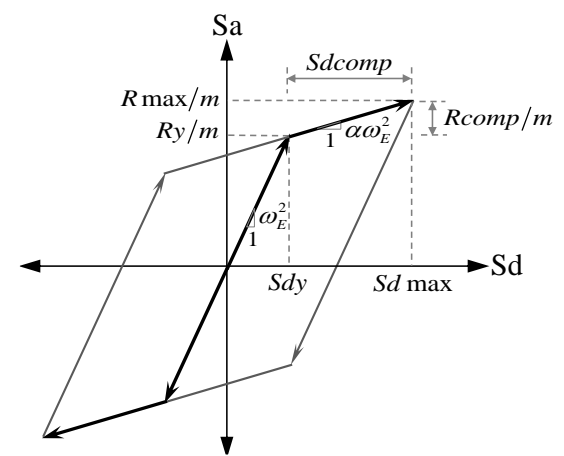

Figura 1. Curva de comportamiento de un oscilador bilineal de $1 \mathrm{GDL}$

Se reconoce que los miembros de concreto reforzado exhiben cierta degradación de rigidez y resistencia cuando se sujetan a fuerzas cíclicas reversibles, por lo que para predecir adecuadamente la respuesta de una estructura, las acciones sísmicas de diseño se deben caracterizar por medio de espectros inelásticos en los que se considere la degradación de estas propiedades. Para realizar el análisis sísmico de estructuras comúnmente se debe suponer que las reglas de histéresis, así como de los parámetros $\alpha$ y de degradación que siguen las relaciones fuerza-desplazamiento de los osciladores de 1GDL que representan a los modos de una estructura de varios grados de libertad (VGDL), son las mismas que siguen las relaciones fuerza-desplazamiento de los miembros estructurales. Con la finalidad de subsanar parcialmente la carencia de esta información, en el presente estudio se desarrolló un procedimiento de análisis sísmico que permite estimar, de manera aproximada, los valores del parámetro ade los osciladores modales, asociados a cierto valor del parámetro $\alpha$ de las relaciones fuerza-desplazamiento de los miembros estructurales. Debido a que no se estimaron los parámetros de degradación que presentan las relaciones fuerza-desplazamiento de los osciladores modales, se considera que éstos no presentan degradación de rigidez y de resistencia. Es evidente, sin embargo, que en la aplicación práctica del procedimiento propuesto se debe considerar el comportamiento degradante de los osciladores.

\section{Principales hipótesis}

La principal hipótesis en que se basa el procedimiento propuesto considera que el comportamiento idealizado del oscilador cuyas propiedades dinámicas corresponden al modo de mayor contribución a la respuesta de la estructura, denominado sistema de referencia de 1GDL (SR1GDL) se puede extrapolar, considerando la contribución de los modos superiores mediante análisis modales espectrales (AME), para 
obtener la también idealizada relación cortante basal-desplazamiento lateral de la azotea, denominada como curva de capacidad (CC) de una estructura de VGDL.

La segunda hipótesis consiste en aceptar como válida la aplicación del AME para calcular la respuesta inelástica de estructuras de VGDL. Las aproximaciones que hacen posible la aplicación del AME a sistemas inelásticos son bien conocidas: (1) ignorar el acoplamiento de las respuestas de los "osciladores modales" debido a la fluencia de la estructura, y (2), considerar aplicable el uso de una regla de combinación modal para superponer las "contribuciones modales" y obtener la respuesta total de la estructura.

En un estudio preliminar Chopra y Goel (2001) desarrollaron un procedimiento de análisis simplificado denominado análisis de la historia dela respuesta modal desacoplada (UMRHA), por sus siglas en inglés, para estudiar el efecto de ignorar el acoplamiento de los modos durante la respuesta inelástica de estructuras. La comparación de las deformaciones laterales de las estructuras estimadas con este procedimiento con las obtenidas por medio de análisis dinámicos inelásticos paso a paso, indica que el efecto del acoplamiento de los modos en la estimación de este parámetro de respuesta no es significativo, incluso para un grado moderado de comportamiento inelástico. Los errores obtenidos son aceptables para fines de análisis y diseño estructural. Más aún, la aplicación de alguna regla de combinación para estimar la respuesta de sistemas inelásticos carece de bases teóricas. Sin embargo, la similitud entre las deformaciones laterales estimadas con el UMRHA y con el denominado "análisis pushover modal" (Chopra y Goel, 2001), el cual ignora el acoplamiento de la misma forma que el UMRHA pero obtiene la respuesta de una estructura mediante una regla de combinación modal, sugiere que el uso de éstas no incrementa la magnitud de los errores debidos a ignorar el acoplamiento de los modos.

\section{Consideraciones y aproximaciones adicionales para implementar el procedimiento de análisis sísmico}

El procedimiento de análisis sísmico propuesto es análogo al considerado en los denominados "pushover adaptables". El análisis inelástico se lleva a cabo en dos etapas, cada una correspondiente a una rama de la CC de la estructura. Los extremos finales de la primera y segunda rama de la CC representan los estados de daño correspondientes al EFI y ELPC, respectivamente. En cada etapa se determinan las denominadas propiedades dinámicas "instantáneas" dela estructura, y en función de éstas y del espectro y/o registro que define la acción sísmica de diseño, se obtienen las fuerzas laterales estáticas equivalentes y las demandas deformación lateral de la estructura en cada etapa. El principal aspecto en que difiere el análisis propuesto de los análisis pushover adaptables, es la forma en la que se determinan las demandas sísmicas de diseño en cada etapa, lo cual se discutirá con detalle posteriormente.

\section{Postulación de una distribución de daño global en la estructura, aceptable para el estado límite de prevención del colapso}

En este trabajo el daño se define por la formación de articulaciones plásticas en las secciones críticas de un miembro estructural, que en el caso de las columnas, son sus secciones extremas. Cuando las acciones sísmicas imponen mayores esfuerzos que las gravitacionales, las secciones críticas de las vigas también son sus secciones extremas, fig. $3 \mathrm{a}$.

Para el análisis y diseño en el ELPC se postula una distribución de daño en la estructura congruente con un estado de daño representativo de este estado límite. Una posible y deseable distribución de daño con esta característica, la cual se muestra en la fig. 2a, corresponde al inicio del mecanismo de colapso que 
buscan inducir los reglamentos mediante los criterios de diseño por capacidad, conocido como mecanismo de columna fuerte-viga débil.

Con base en resultados de estudios analíticos, algunos autores (Park y Paulay, 1975; Sullivan et al., 2008), muestran que durante la respuesta de estructuras reticulares de baja altura, en las cuales comúnmente rige la contribución del modo fundamental, puede haber instantes en los que las articulaciones plásticas de todas las vigas se desarrollen simultáneamente. En estructuras de mayor altura, en las que usualmente la contribución de los modos superiores es más significativa, las articulaciones en vigas se desarrollan por grupos de entrepisos. Cabe mencionar, sin embargo, que el desarrollo de las articulaciones en todas las vigas de un entrepiso dado solo será posible si la máxima distorsión de dicho entrepiso es mayor que la asociada a su fluencia incipiente $\left(\gamma_{f f l u e n c i a}\right)$. Esta situación se ilustra en la fig. $2 \mathrm{~b}$, donde se muestra un típico perfil de las máximas distorsiones de entrepiso en el ELPC de un marco rígido regular en altura y en distribución de masas, en el que todas sus vigas tienen el mismo peralte. Se observa que las vigas en las que no se desarrollarían articulaciones plásticas, son las ubicadas en los entrepisos en los que su distorsión es menor al valor correspondiente a fluencia incipiente.

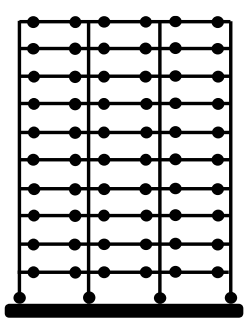

a)
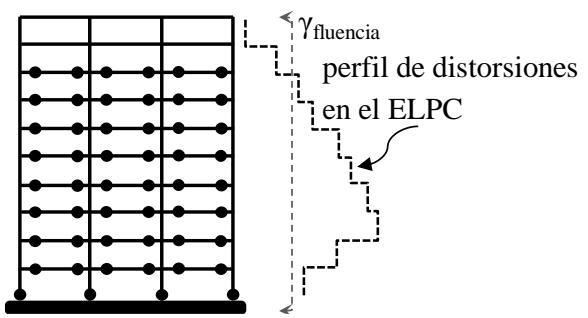

b)

Figura 2. a). Distribución de daño representativa de la formación del mecanismo de colapso columna fuerte-viga débil y b) Condición en la que las vigas de ciertos entrepisos no deben fluir

En este trabajo se establece la aproximación de que independientemente de la magnitud de contribución de los modos superiores, todas las articulaciones plásticas, tanto en las vigas como en las columnas que deban de fluir, se desarrollarán simultáneamente. La correspondiente distribución de daño caracterizará la mínima rigidez lateral posible que una estructura presentará durante su repuesta, por lo que esta aproximación permite formular un modelo analítico para determinar su máxima respuesta de desplazamiento.

Al aplicar esta aproximación en estructuras en las que la contribución del modo fundamental sea predominante, es posible que se caracterice adecuadamente su rigidez lateral en la máxima respuesta de desplazamiento. En estructuras reticulares hasta de mediana altura, para las cuales se ha determinado que aún es económicamente factible el uso de este sistema estructural (García et al., 1996), se espera que al aplicar esta aproximación se obtenga un límite inferior razonable de su rigidez lateral en su máxima respuesta, con lo que se puede obtener un límite superior aceptable de sus deformaciones laterales máximas.

\section{Modelación simplificada del daño estructural}

La inelasticidad a lo largo de un elemento estructural se representa de forma análoga al conocido modelo del elemento discreto (Otani, 2002), en el cual el elemento se subdivide en segmentos de línea, a cada uno de los cuales se le asigna un comportamiento histerético fuerza-desplazamiento elástico lineal o inelástico. En el presente estudio, los elementos estructurales se dividen en tres segmentos de línea. 
Los segmentos extremos representan las articulaciones plásticas, las cuales se caracterizan de manera simplificada mediante: (1), una longitud equivalente de plastificación $(l p)$, cuyo valor se toma de manera conservadora como $h / 2$, donde h es el peralte de la sección. (2), cierta rigidez de posfluencia a flexión constante a lo largo de $l p$, expresada en función de una fracción $\beta$, de la rigidez a flexión efectiva inicial del elemento estructural $\left(E I_{\text {eff }}\right)$. E es el módulo de elasticidad del concreto $e I_{\text {eff }}$ es el momento de inercia efectivo del elemento estructural que presenta en el EFI.

El segmento central representa la porción del miembro que permanece elástica y se le asigna una $E I_{\text {eff }}$ constante en toda su longitud. En la fig. 3a se muestra un esquema del modelo de daño simplificado propuesto. De acuerdo con un estudio paramétrico en secciones de vigas y columnas de concreto reforzado realizado por los autores (Barradas, 2013), el valor de $\beta$ obtenido a partir de diagramas momento-curvatura de sus secciones varía entre 1\% y $4 \%$. Para vigas y columnas se sugiere usar $2 \%$ y $4 \%$, respectivamente, valores que pueden ser suficientemente aproximados para fines prácticos; sin embargo, en la implementación del procedimiento se pueden usar los que se consideren más adecuados.

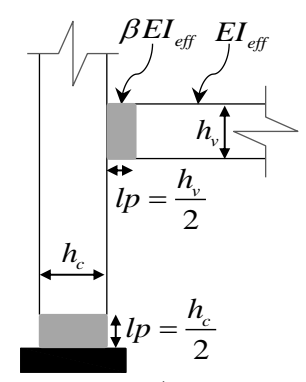

a)

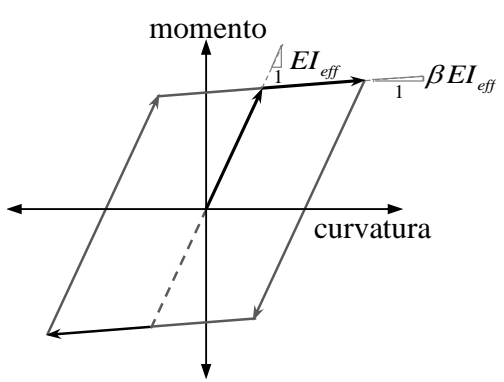

b)

Figura 3. Modelo elástico aproximado para representar la inelasticidad en las secciones críticas de los elementos estructurales

En el modelo analítico de la estructura para la primera etapa del análisis se asigna una rigidez $E I_{\text {eff }}$ a todas las secciones de los elementos estructurales, mientras que en el modelo analítico para la segunda etapa, la rigidez a lo largo de los elementos se define de acuerdo al modelo simplificado discutido en la presente sección. Mediante estas consideraciones se busca representar en las secciones críticas un comportamiento cíclico idealizado ante cargas reversibles que no presenta degradación de rigidez ni de resistencia, como se muestra en la fig. 3 b.

\section{Necesidad de diseñar las columnas por capacidad}

Debido a que en el modelo elástico simplificado de la estructura para el análisis sísmico en el ELPC se considera que todas las articulaciones plásticas se desarrollan simultáneamente, no se pueden considerar algunos aspectos del comportamiento dinámico de estructuras debidos al desarrollo gradual de las articulaciones. Entre estos aspectos se encuentran el cambio instantáneo de sus propiedades dinámicas y la correspondiente distribución de las fuerzas de inercia, así como la redistribución de los momentos flexionantes de las vigas y columnas. Como consecuencia, se subestiman de ligera a significativamente, las resistencias a flexión y a cortante de las columnas necesarias para garantizar que su comportamiento ante la acción sísmica correspondiente al ELPC sea elástico.

Para reducir la posibilidad de que se desarrollen articulaciones plásticas en los extremos de todas las columnas a excepción de los extremos inferiores de las columnas de la base, sus resistencias de diseño se deben calcular aplicando los criterios de diseño por capacidad que indican los reglamentos. Estos criterios se han desarrollado para su implementación en el procedimiento de diseño sísmico basado en fuerzas, el 
cual considera que el comportamiento global ante fuerzas laterales de una estructura es elastoplástico. Debido a esto, las fuerzas internas a considerar para aplicar los criterios de diseño por capacidad, son las obtenidas en la primera etapa del análisis.

\section{Aproximación de la resistencia de fluencia y ductilidad de desplazamiento de los modos superiores}

En el procedimiento de análisis, al definir las propiedades y parámetros de respuesta del SR1GDL, paralelamente se definen los correspondientes de los modos superiores. Para determinar los parámetros de respuesta $R$ max/m y $S d m a x$ de los osciladores que representan a los modos superiores, es necesario determinar primero su ductilidad de desplazamiento, $\mu$, la cual es función de sus propiedades $\alpha$ y $R y / m$. En la fig. 4a se muestra la típica tendencia creciente del parámetro $\alpha$ con los modos de vibrar de una estructura en la que se propuso una distribución de daño como la mostrada en la fig. 2a. Esta tendencia indica que los modos superiores presentan una mayor rigidez de posfluencia que el modo fundamental.

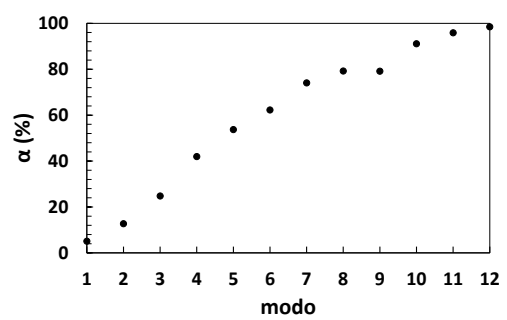

a). Variación típica del parámetro $\alpha$ en los osciladores modales de una estructura de VGDL

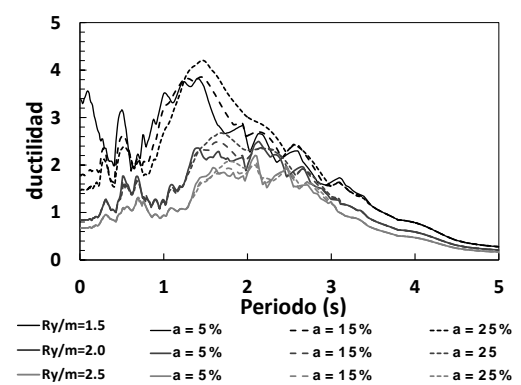

b). Espectros de resistencia constante para un sitio con suelo blando en el Valle de México

Figura 4. Parámetros que influyen en la resistencia y ductilidad de osciladores de 1GDL

Por medio de estudios analíticos se ha mostrado (Rodríguez et. al 2002; Sullivan et al., 2008) que la respuesta de los modos superiores es afectada en menor medida por el desarrollo de inelasticidad (ductilidad), que la respuesta del modo fundamental.

Para obtener una aproximación de la $R y / m$ y por tanto de la $\mu$ de los modos superiores, en el presente estudio se acepta, en principio, que estos modos han de desarrollar ductilidades menores a la del modo fundamental, por lo que la $R y / m$ de dichos modos debe ser como mínimo, la requerida para desarrollar la ductilidad del SR1GDL. Sin embargo, al tener estos modos una mayor rigidez de posfluencia y dependiendo de la región espectral en la que se ubiquen sus periodos, desarrollarán ductilidades menores, similares o incluso mayores a la del SR1GDL, como a continuación se discute en referencia a la fig. $4 \mathrm{~b}$. En esta figura se muestran tres grupos de espectros con $R y / m$ constantes e iguales a $1.5,2.0$ y $2.5 \mathrm{~m} / \mathrm{s} 2$ para valores del parámetro $\alpha$ de $5 \%, 15 \%$ y $25 \%$. Cabe mencionar que para el cálculo de estos espectros se consideró que los osciladores no presentan degradación de rigidez ni de resistencia. Además, estos espectros corresponden a un registro en suelo blando del Valle de México. Cabe mencionar, sin embargo, que dichos espectros presentan características similares en suelos con distintos grados de firmeza.

En estos espectros se pueden distinguir tres regiones: En la primera región comprendida de 0.0 a aproximadamente $1.3 \mathrm{~s}$, entre mayor rigidez de posfluencia posea un oscilador con una $R y / m$ dada, su demanda de ductilidad será, en general, menor. En la segunda región comprendida entre 1.3 y $3.5 \mathrm{~s}$, por el contrario, entre mayor rigidez de posfluencia posea un oscilador, su demanda de ductilidad será, en 
general, mayor. En la tercera región, comprendida de $3.5 \mathrm{~s}$ en adelante, independientemente de la rigidez de posfluencia que tenga el oscilador, desarrollará prácticamente la misma ductilidad. Además de las observaciones anteriores se pueden hacer las siguientes: (1) No existe una tendencia claramente consistente en las ductilidades que se puedan desarrollar en las dos primeras regiones espectrales, ya que dentro de ellas, existen subregiones en las que se presentan tendencias contrarias. (2), Conforme se incrementa $R y / m$, menores son las diferencias en las ductilidades desarrolladas por osciladores con distintos valores de $\alpha$. (3), El periodo exacto que delimita estas regiones depende del coeficiente de resistencia, y el periodo que delimita las dos primeras regiones comúnmente es menor que el periodo dominante del movimiento del terreno.

En la aplicación práctica del procedimiento propuesto mediante el uso de espectros inelásticos de diseño, para calcular la ductilidad de los modos superiores a partir de su $R y / m$ y $\alpha$ conocidos, se pueden usar las relaciones de desplazamiento inelástico $\left(C_{R}\right)$ desarrolladas por Ruiz-García y Miranda (2003 y 2006). $C_{R}$ se define como el desplazamiento inelástico máximo $\left(\Delta_{\text {inelastico }}\right)$, dividido entre el desplazamiento elástico máximo, $S d$, de un oscilador de $1 \mathrm{GDL}$, ec. 1:

$C_{R}=\frac{\Delta_{\text {inelastico }}}{S d}$

En la ecuación anterior $\Delta_{\text {inelastico }}$ se calcula en osciladores con una relación $(R)$ constante de resistencia requerida para mantener al oscilador elástico $(m S a)$, a resistencia de fluencia $(F y)$, es decir, ec. 2:

$$
R=\frac{m S a}{F y}
$$

donde $m$ y $S a$ son, respectivamente, la masa y la aceleración espectral elástica del oscilador.

Una vez calculado $C_{R}$ en función de $R$ con ayuda de las gráficas o ecuaciones que se presentan en

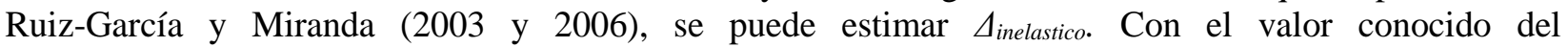
desplazamiento espectral de fluencia $\left(\Delta_{y}\right)$ se calcula la ductilidad del oscilador. Mediante la ec.3 tomada de Ruiz-García y Miranda (2006), se puede determinar el valor de $C_{R}$

$$
C_{R}=\theta_{1}+(R-1)\left[\frac{1}{\theta_{2}\left(T / T_{g}\right)}\right]+\theta_{3}\left(T / T_{g}\right) \exp \left\{-4.5\left[\ln \left(T / T_{g}-0.05\right)\right]^{2}\right\}+\theta_{4}\left(T / T_{g}\right) \exp \left\{\theta_{5}\left[\ln \left(T / T_{g}+0.67\right)\right]^{2}\right\}
$$

donde $T$ es el periodo de vibración, $T g$ es el periodo dominante del movimiento del terreno y $\theta_{1}, \theta_{2}, \theta_{3}, \theta_{4}, \theta_{5}$, son parámetros cuyos valores dependen del tipo de suelo, y se muestran en la tabla 1.

Tabla 1. Parámetros estimados para la ecuación 4, obtenidos del valor medio de $C_{R}$ para el conjunto de registros en la ciudad de México, considerando comportamiento elastoplástico

\begin{tabular}{cccccc}
\hline Conjunto ciudad de México & $R=2.0$ & $R=3.0$ & $R=4.0$ & $R=5.0$ & $R=6.0$ \\
\hline$\theta_{1}$ & 1.096 & 1.104 & 1.119 & 1.148 & 1.149 \\
$\theta_{2}$ & 3.685 & 4.489 & 5.674 & 7.083 & 8.608 \\
$\theta_{3}$ & -0.640 & -1.023 & -1.197 & -1.307 & -1.341 \\
$\theta_{4}$ & -0.589 & 0.578 & 0.562 & 0.537 & 0.522 \\
$\theta_{5}$ & -14.914 & -52.803 & -132.146 & -177.709 & -211.622 \\
\hline
\end{tabular}




\section{DESCRIPCIÓN DEL PROCEDIMIENTO}

El procedimiento de diseño consiste en construir la CC de la estructura para la cual se satisface el $\mathrm{OD}$ considerado. La rama elástica inicial de la $\mathrm{CC}$ se define por la rigidez lateral global y la resistencia requerida para que los componentes estructurales y no estructurales del edificio no presenten daño ante la acción sísmica de diseño asociada al ELS. La ductilidad global de desplazamiento de la estructura es la requerida para que, dadas su resistencia de fluencia y rigidez de posfluencia correspondiente a un estado de daño postulado para el ELPC, se desarrolle una distorsión de entrepiso máxima menor o igual a la permisible para este estado límite.

\section{Alcances del procedimiento}

En la etapa de desarrollo actual, el procedimiento solo puede aplicarse para el diseño estructuras bidimensionales conformadas por marcos rígidos de concreto reforzado, en las que se pueda ignorar los efectos de la interacción suelo estructura.

\section{Diseño para el Estado Límite de Servicio}

El criterio de desempeño para este estado límite es que la estructura y sus componentes no estructurales no presenten daño ante la acción sísmica de diseño correspondiente. El control del daño estructural requiere que la respuesta de la estructura sea elástica, mientras que el control del daño no estructural se logra limitando la máxima distorsión de entrepiso $\left(\gamma_{\max }\right)$ a la permisible para el ELS $\left(\gamma_{E L S)}\right.$. El procedimiento inicia dimensionando las secciones transversales de los elementos para proporcionarle a la estructura la rigidez lateral necesaria para que $\gamma_{\max }$ sea aproximadamente igual a $\gamma_{E L S}$. Las dimensiones y relaciones de aspecto de las secciones deben estar dentro de los intervalos establecidos por el reglamento. Para considerar la pérdida de rigidez en los miembros debida al agrietamiento se recomienda usar en vigas: $I_{e f f}=0.7 I_{g}$ y en columnas: $I_{e f f}=I_{g}$, donde $I_{g}$ es el momento de inercia de la sección transversal bruta de concreto. El dimensionamiento se lleva a cabo de forma iterativa mediante una serie de AME, considerando el espectro elástico representativo de la acción sísmica de diseño correspondiente al ELS. El proceso iterativo termina cuando $\gamma_{\max }$ es aproximadamente igual a $\gamma_{E L S}$.

\section{Caracterización preliminar del estado de fluencia estructural incipiente.}

Estudios recientes (Priestley, 1998), muestran que una vez definidas la configuración geométrica y las dimensiones de los miembros de estructuras de concreto reforzado, queda definido también un valor aproximado de la distorsión de entrepiso correspondiente a la fluencia incipiente del sistema. Dicho valor está dado por la ec. 4, la cual es una expresión semi-empírica propuesta por Priestley (1998), cuyos resultados mostraron buena correlación con los de pruebas experimentales.

Debido a que en el ELPC se fomenta el desarrollo del mecanismo de colapso columna fuerte-viga débil, en el estado límite de fluencia incipiente solo las vigas estarán en dicha condición, por lo que presentarán una pérdida de rigidez mayor a la estimada en condiciones de servicio. Para considerar dicha pérdida se recomienda usar $I_{e f f}=0.5 I_{g}$, caracterizar nuevamente el modelo analítico de la estructura y realizar un análisis de valores característicos para obtener sus nuevas propiedades dinámicas.

Para el diseño de la estructura en este estado se debe disponer de su correspondiente acción sísmica caracterizada por un espectro de diseño elástico. Sin embargo, puesto que en los actuales reglamentos comúnmente no se estipula esta demanda sísmica, se recomienda el siguiente procedimiento: 
Se obtiene una estimación preliminar de la configuración de distorsiones de entrepiso correspondiente a este estado, considerando solo la contribución del modo fundamental. Para este fin, del análisis de valores característicos previamente realizado se toma el vector correspondiente a la forma del modo fundamental, que define la configuración de los desplazamientos laterales correspondientes a dicho modo. De esta configuración se obtiene $\gamma_{\max }$. Posteriormente se calcula la distorsión de fluencia $\left(\gamma_{y}\right)$ en el entrepiso donde se presentó $\gamma_{\max }$ mediante la ec. 4:

$\gamma_{y}=\frac{0.30 \varepsilon_{y} l_{b}}{h_{b}}=$

dónde: $\varepsilon_{y}$, es la deformación unitaria a tensión del acero de refuerzo de las vigas, $h_{b}$ es su peralte total y $l_{b}$ su longitud eje a eje de columnas.

Los desplazamientos laterales de los niveles se escalan en una cantidad, $f e$, tal que $\gamma_{\max }$ sea igual a $\gamma_{y}$. A partir del desplazamiento escalado en la azotea ( $\Delta^{a z o t e a} f e$ ) se obtiene el desplazamiento de fluencia del SR1GDL $\left(S d_{y l}\right)$ mediante la ec.5.

$S d_{y 1}=\frac{\Delta^{\text {azotea }} f e}{F P_{1}^{\text {catea }}}$

donde $F P^{l \text { azotea }}$ es el producto del factor de participación del modo fundamental de la estructura, por la amplitud de su forma modal en la azotea.

Cabe notar que con la ec. 4 se obtiene un valor total de la distorsión, por lo que la aplicación de esta ecuación es justificable solo sien el entrepiso donde se presenta $\gamma_{\max }$ la contribución de los modos superiores no es significativa (como criterio, que incremente la distorsión en menos de 10\%).Si se espera que esta condición no se cumpla, es recomendable establecer un dimensionamiento de las vigas y columnas de tal forma que en el estado elástico de la estructura su máxima distorsión se ubique en los entrepisos inferiores, en los cuales la contribución de dichos modos es menor. Se recomienda además aplicar la ec. 5 en el primer nivel, considerando $F P_{l}^{\text {nivel } 1}$ y $\Delta^{\text {nivel } l}$.

Una vez calculado el valor de $S d_{y l}$ se obtiene su correspondiente aceleración espectral ( $\left.S a_{y l}\right)$ mediante la ec. 6 .

$S a_{y 1}=\omega_{1 E}^{2} S d_{y}$

donde $\omega_{I E}$ es la frecuencia natural del modo fundamental de la estructura en el estado de fluencia incipiente.

La relación expresada en la ec. 6 se basa en la suposición de que la señal es armónica simple, sin embargo, los errores debidos a dicha suposición generalmente no son significativos (FIB-2003).

\section{Diseño para el Estado Límite de Prevención del Colapso}

La mayoría de los reglamentos de diseño sísmico internacionales, incluido el RCDF-2004, estipulan que para que una estructura presente un razonable margen de seguridad contra el colapso se le debe proporcionar: (1) una resistencia de fluencia talque su máxima demanda de ductilidad global sea menor o 
igual a su capacidad dúctil. (2), una rigidez lateral suficiente para limitar la máxima distorsión de entrepiso inelástica a un valor permisible. El procedimiento a seguir para el diseño en este estado límite es el siguiente:

Se propone una distribución de daño factible de presentarse en la estructura, para lo cual es necesario determinar si la distorsión de cada uno de los entrepisos será mayor que su correspondiente $\gamma_{y}$. Para este efecto, en un primer tanteo se propone una distribución de daño como la mostrada en la fig. 2a, y a partir de ésta se formula un modelo elástico de la estructura. Se le efectúa un análisis de valores característicos para obtener algunas propiedades dinámicas correspondientes al modo fundamental: periodo en su estado dañado $\left(T_{I D}\right)$, configuración de desplazamientos laterales de los niveles y la máxima distorsión correspondiente a dicha configuración, $\left(\gamma_{1 \max }\right)$. Con los periodos del modo fundamental de la estructura en sus estados de fluencia incipiente $\left(T_{I E}\right)$ y con daño, $T_{I D}$, se calcula el parámetro $\alpha$ correspondiente al SR1GDL mediante la ec. 7:

$$
\alpha_{1}=\left(\frac{T_{1 E}}{T_{1 D}}\right)^{2}
$$

Se propone un valor preliminar de la máxima distorsión correspondiente al modo fundamental $\left(\gamma_{E L P C I}\right)$, cercano al máximo permisible indicado por el reglamento, y los desplazamientos de los niveles obtenidos en el paso anterior se escalan en una cantidad, $f e$, tal que el valor de $\gamma_{\max I}$ sea igual a $\gamma_{E L P C I}$.A continuación se comparan las distorsiones estimadas para cada uno de los entrepisos con sus correspondientes $\gamma_{y}$. Si la distorsión de algún entrepiso es menor a su $\gamma_{y}$, no fluirán las vigas, por lo que no se debe considerar daño en ellas y se tendrá que modificar la distribución de daño propuesta. La definición de una distribución de daño factible puede requerir varias iteraciones.

Una vez definida la distribución de daño, a partir del desplazamiento escalado en la azotea $\left(\triangle_{1}{ }^{\text {azotea }} f e\right)$ se obtiene el desplazamiento del SR1GDL correspondiente al ELPC $\left(S d_{E L P C I}\right)$ mediante la ec. 8. El cociente de este desplazamiento entre $S d_{y l}$ define la demanda de ductilidad $\left(\mu_{1}\right)$ del SR1GDL.

$$
S d_{E L P C}=\frac{\Delta^{\text {azotea }} f e}{F P_{1}^{\text {azotea }}}
$$

A partir del espectro elástico que caracteriza el nivel de diseño sísmico correspondiente al ELPC, se calcula su espectro inelástico de resistencia por unidad de masa correspondiente a los valores de $\mu_{1}, \alpha_{1}$, y a una fracción de amortiguamiento crítico, $\xi=5 \%$. En este espectro se lee la ordenada correspondiente a $T_{I E}$, $\left(R y / m_{l}\right)$. Si $R y / m_{l}$ presenta una diferencia de hasta $\pm 5 \%$ con respecto a $S a_{y}$, el valor de $\mu_{l}$ se considera apropiado. Si la diferencia es mayor, se deberá proponer otro valor de $\gamma_{E L P C l}$, con la finalidad de adecuar $\mu_{1}$, de tal forma que $R y / m_{l}$ sea aproximadamente igual a $S a_{y}$.

En las distorsiones de entrepiso que se obtienen en esta etapa se considera únicamente la contribución del modo fundamental. Al final del procedimiento, cuando se considera la contribución de todos los modos, se deberá revisar si las distorsiones de los entrepisos que se consideraron no fluirían son mayores a sus correspondientes $\gamma_{y}$. En este caso se deberá modificar la distribución de daño propuesta e iniciar esta etapa del diseño.

La aceleración del SR1GDL correspondiente al ELPC $\left(S a_{E L P C}\right)$ se obtiene por medio de la ec. 9. En esta etapa están definidos todos los parámetros de respuesta de la curva de comportamiento del SR1GDL. 
$S a_{E L P C}=S a_{y 1}\left[1+\alpha_{1}\left(\mu_{1}-1\right)\right]$

\section{Caracterización definitiva del estado de fluencia estructural incipiente}

Conocidos los parámetros $\mu_{1}$ y $\alpha_{l}$, se caracteriza el estado de fluencia incipiente de la estructura. Para esto se desarrolla un AME considerando el espectro inelástico de resistencia previamente calculado, sus propiedades de rigidez correspondientes a fluencia incipiente y las cargas gravitacionales actuantes, fig. 5a. De este análisis se obtienen los parámetros de respuesta correspondientes a la fluencia incipiente de la estructura: desplazamientos laterales de sus niveles y distorsiones de entrepiso, resistencias de diseño de las vigas y columnas que deben fluir, así como parte de la resistencia de las vigas que no deben fluir. Se obtienen también las resistencias básicas para la aplicación del procedimiento de diseño por capacidad para las columnas en las que no deben desarrollarse articulaciones plásticas. De este análisis quedan definidas las primeras ramas de las curvas de comportamiento modales y la primera rama de la curva de capacidad.

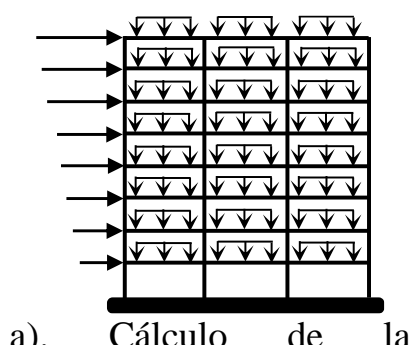

a). Cálculo de la respuesta correspondiente a fluencia incipiente

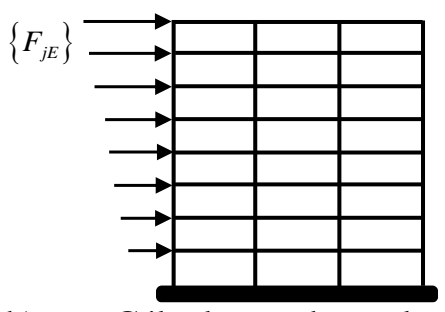

b). Cálculo de la c) contribución del modo $j$ a la respuesta elástica máxima de la estructura.

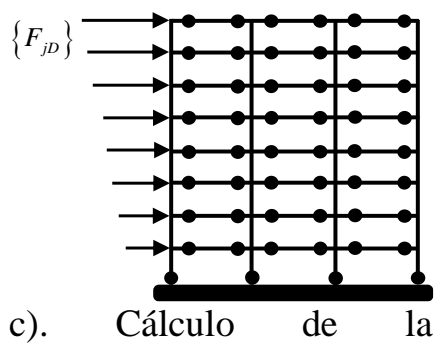

contribución del modo $j$ a

la respuesta

complementaria de la estructura.

Figura 5. Modelo estructurales para el cálculo de la respuesta elástica y complementaria de una estructura

\section{Caracterización de la curva de capacidad de la estructura}

La contribución del modo $j$ a los parámetros de interés de la respuesta elástica máxima de la estructura, por ejemplo, al cortante basal de fluencia $\left(V b y_{j}\right)$ y al desplazamiento lateral de la azotea asociado $\left(\Delta y_{j}^{a z o t e a}\right)$, se obtienen de analizar la estructura sin cargas gravitacionales y con propiedades de rigidez correspondientes a fluencia incipiente. En análisis se lleva a cabo para un patrón de fuerzas laterales estáticas equivalentes asociadas a dicho modo, fig. 5b, definido por el siguiente vector, ec. 10.

$$
\left\{F_{j E}\right\}=\Gamma_{j E}\left\{\varphi_{j E}\right\}[m] R y / m_{j}
$$

donde: $\Gamma_{j E} \mathrm{y}\left\{\varphi_{j E}\right\}$ son el factor de participación y el vector de amplitudes del modo $j$ de la estructura en su estado de fluencia incipiente, $[\mathrm{m}]$ es la matriz de masa y $R y / m_{j}$ es la resistencia de fluencia por unidad de masa del modo $j$ leída del espectro inelástico calculado para los valores de $\mu_{1}$ y $\alpha_{1}$.

A continuación se calcula la contribución del modo $j$ a estos parámetros de la respuesta complementaria de la estructura: cortante basal complementario $\left(V b c o m p_{j}\right)$ y el desplazamiento lateral de la azotea asociado $\left(\triangle c o m p_{j}^{a z o t e a}\right)$, procediendo de la siguiente manera. A partir de las $R y / m_{j}$ definidas en el paso anterior y los correspondientes valores del parámetro $\alpha_{j}$, calculados en forma análoga a la del SR1GDL, se determinan sus ductilidades de desplazamiento, $\mu_{j}$, recurriendo al uso de las ecs. 1,2 y 3 . Con 
el conjunto de parámetros previamente calculados se determinan las resistencias complementarias por unidad de masa mediante la ec. 11:

$R$ comp $/ m_{j}=R y / m_{j} \alpha_{j}\left(\mu_{j}-1\right)$

Con estas resistencias se calcula para cada modo en su estado dañado, un patrón de fuerzas laterales estáticas equivalentes definido por el siguiente vector, ec. 12,

$\left\{F_{j D}\right\}=\Gamma_{j D}\left\{\varphi_{j D}\right\}[m] R y / m_{j}$

donde: $\Gamma_{j D} y\left\{\varphi_{j D}\right\}$ son respectivamente, el factor de participación y el vector de amplitudes del modo $j$ de la estructura en su estado dañado.

Con cada vector de fuerzas se realiza un análisis estático a la estructura con la distribución de daño postulada, y sin las cargas gravitacionales, como se muestra en la fig. 5c.

Se calcula la contribución del modo $j$ a estos parámetros de la respuesta inelástica máxima de la estructura: cortante basal máximo $\left(\operatorname{Vbmax}_{j}\right)$ y el desplazamiento lateral de la azotea asociado ( $\left.\triangle \max _{j}{ }^{\text {azotea }}\right)$, sumando las contribuciones modales obtenidas en los análisis anteriores, figs. 5b y 5c.

Finalmente, el valor total de estos parámetros de la respuesta inelástica máxima de la estructura: cortante basal máximo (Vbmax), y el desplazamiento lateral de la azotea asociado ( $\Delta$ max $^{a z o t e a}$ ), se obtienen superponiendo las contribuciones de todos los modos obtenidas en el paso anterior. Si se considera que la regla de combinación modal de la raíz cuadrada de la suma de los cuadrados es apropiada, el valor de Vbmax se calculará de acuerdo a lo expresado en la ec. 13.

$V b_{\max } \approx \sqrt{\sum_{j=1}^{n \bmod o s}\left(V b y_{j}+V b c o m p_{j}\right)^{2}}$

La máxima distorsión de entrepiso resultante será la de diseño para el ELPC ( $\left.\gamma_{E L P C}\right)$. Mediante el diseño estructural de las secciones se verifica si éstas son adecuadas para albergar la cantidad de refuerzo requerida cumpliendo con los requisitos reglamentarios pertinentes. En caso de no ser posible, se deberán modificar las dimensiones de las secciones, lo que implicaría iniciar el procedimiento.

En la fig.6 se muestra un diagrama de flujo del procedimiento de diseño propuesto. 


\section{Diseño para el estado límite de servicio (ELS)}

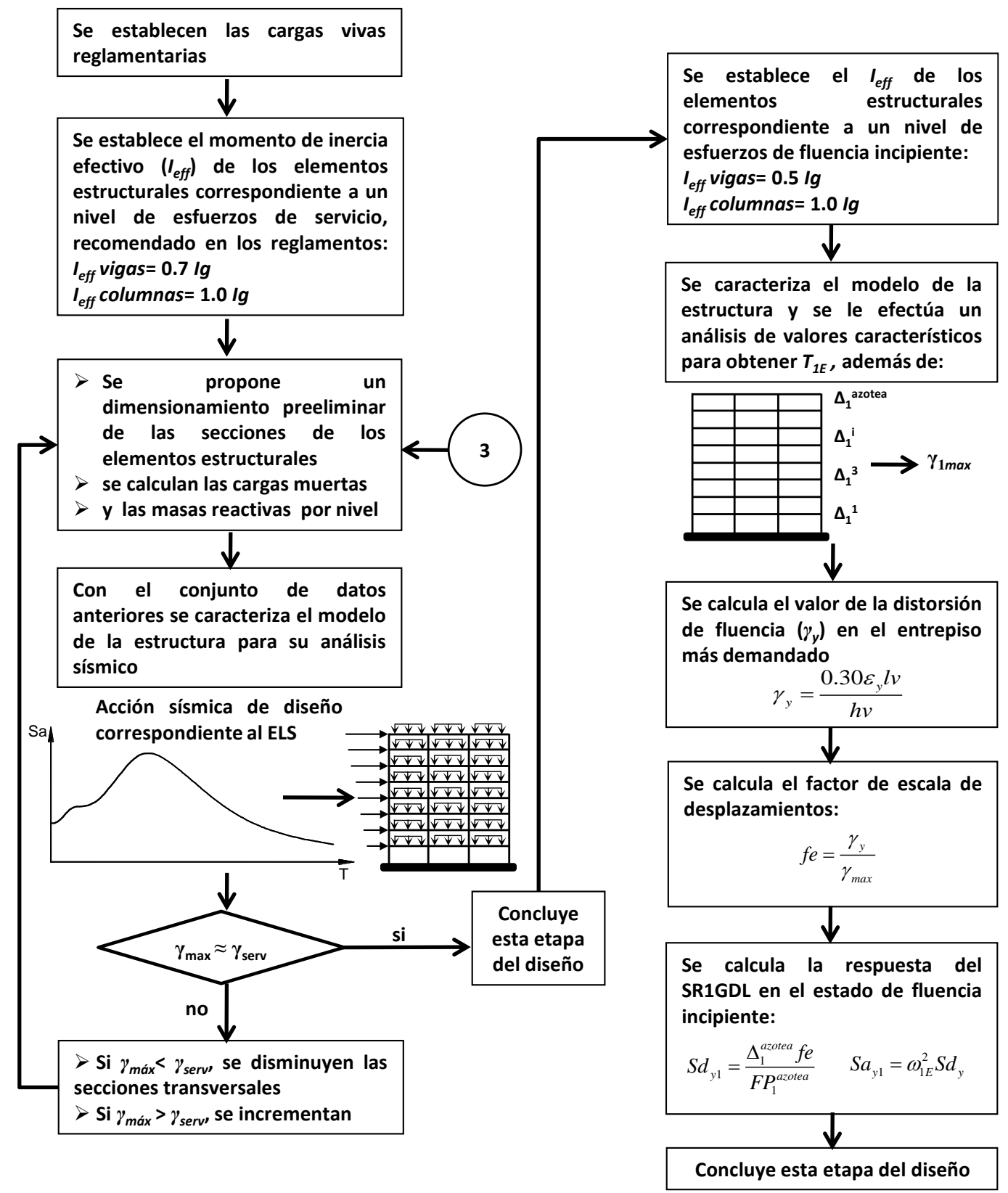

(a) 


\section{Diseño para el estado límite de prevención del colapso (ELPC)}
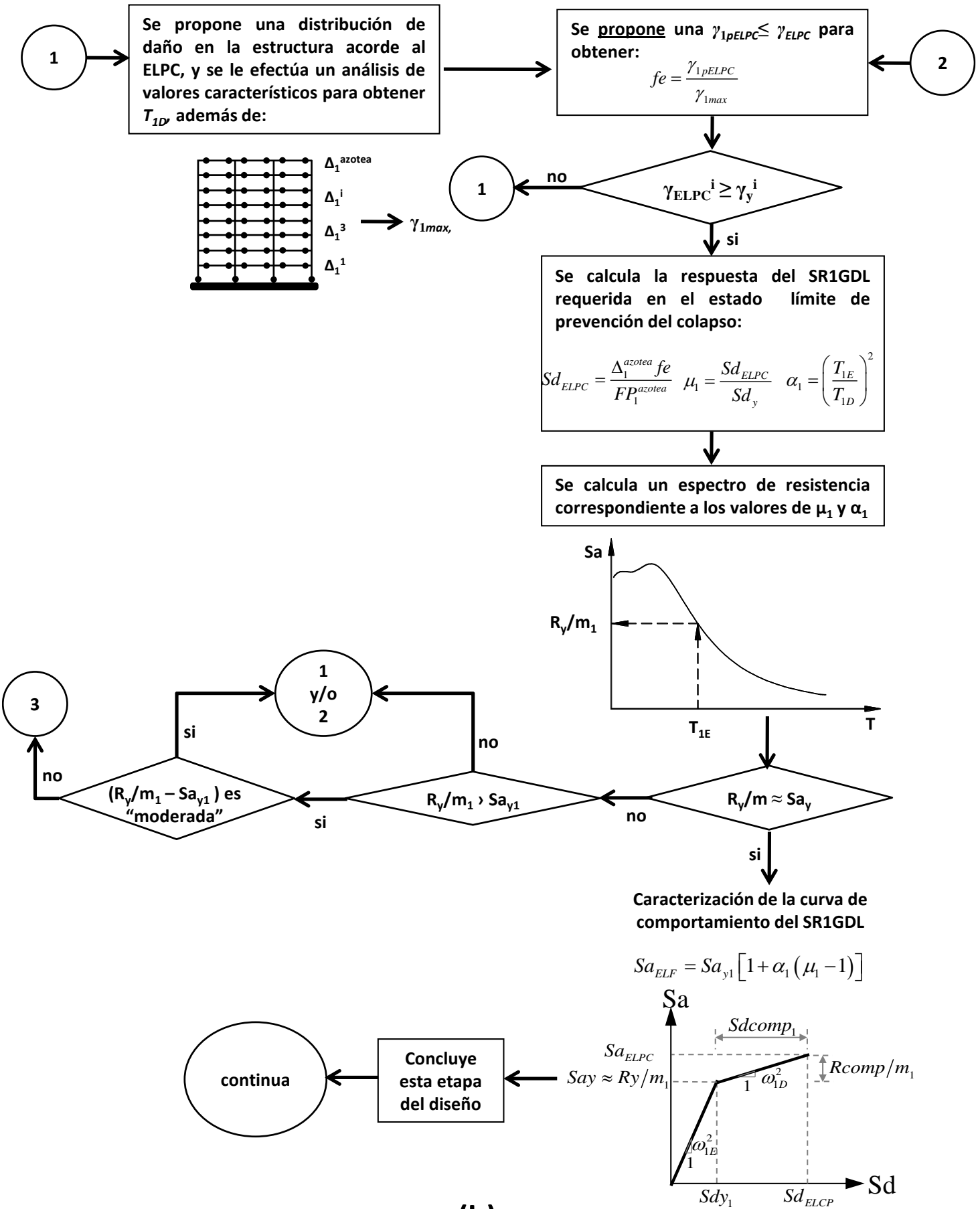

(b) 


\section{Caracterización de la curva de capacidad de la estructura}

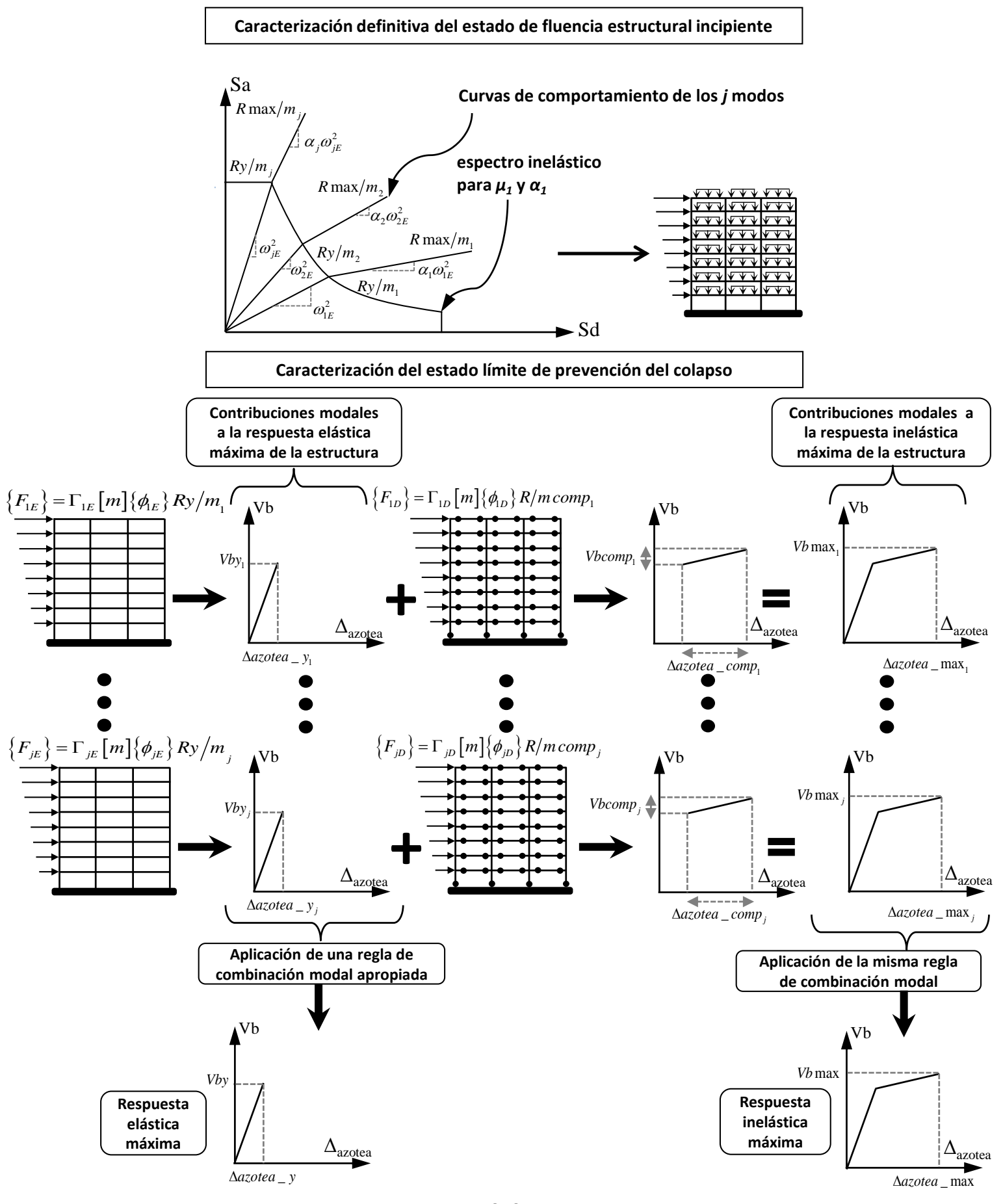

(c)

Figura 6. Diagrama de flujo del procedimiento propuesto 


\section{EJEMPLOS DE APLICACIÓN}

Para ilustrar la implementación del procedimiento propuesto y mostrar su eficacia, en esta sección se presentan algunos ejemplos de aplicación y validación del mismo. Los resultados mostrados en estos ejemplos son representativos de los obtenidos de un mayor número de ejemplos realizados durante el desarrollo conceptual y numérico del procedimiento propuesto, los cuales se presentan en Barradas (2013).

\section{Modelos estructurales considerados}

Se diseñan las estructuras cuyas configuraciones geométricas se muestran en la fig. 7. Se trata de marcos momento-resistentes de concreto reforzado con un sistema de piso a base de losas macizas construidas monolíticamente con las trabes de soporte. Dos marcos son verticalmente regulares, uno de 17 niveles y otro de 8 , y representan estructuras de moderada y baja altura, respectivamente. Los otros dos marcos son de 12 niveles y verticalmente irregulares, y representan estructuras de moderada altura. Uno de estos marcos representa un típico caso práctico, conocido como estructura con el primer entrepiso "suave", el cual al tener una altura considerablemente mayor a los entrepisos superiores, presenta un rigidez lateral menor.

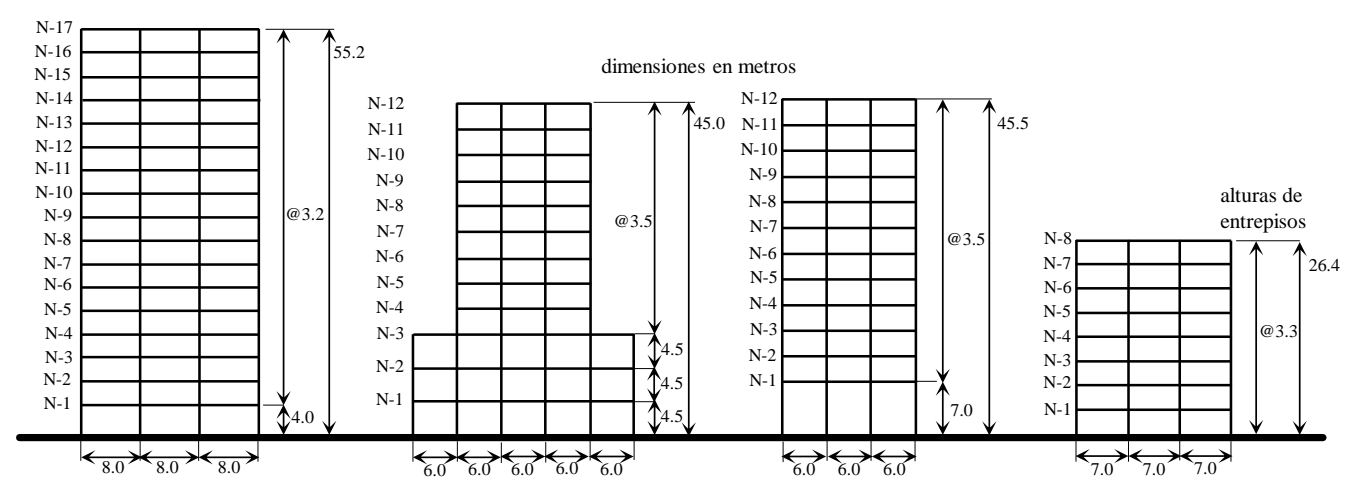

Figura 7. Configuraciones geométricas de los marcos a diseñar

Las cargas gravitacionales de diseño muertas y vivas instantáneas consideradas son las estipuladas en el RCDF-2004 para uso de oficinas. La carga muerta total y viva instantánea en entrepisos son 630 $\mathrm{kgf} / \mathrm{m}^{2}$ y $180 \mathrm{kgf} / \mathrm{m}^{2}$, respectivamente. La carga muerta total y viva instantánea en azotea son $455 \mathrm{kgf} / \mathrm{m}^{2}, \mathrm{y}$ $70 \mathrm{kgf} / \mathrm{m}^{2}$, respectivamente. El área tributaria de los elementos estructurales se obtuvo considerando que las crujías perpendiculares a los marcos tienen la misma longitud. Las propiedades mecánicas de los materiales usados son las recomendadas por el RCDF-2004 para el diseño sísmico de estructuras dúctiles, y son: concreto con un peso volumétrico de $2400 \mathrm{kgf} / \mathrm{m}^{3}$ y resistencia a la compresión de $250 \mathrm{kgf} / \mathrm{cm}^{2}$ y acero de refuerzo con un esfuerzo de fluencia de $4200 \mathrm{kgf} / \mathrm{cm}^{2}$.

Los índices de desempeño estipulados por el RCDF-2004 para estructuras conformadas por marcos de concreto reforzado son: para el ELS distorsiones de entrepiso comprendidas entre 0.2 y $0.4 \%$., para el ELPC: distorsiones de entrepiso comprendidas entre $1.5 \%$ y $3 \%$, y valores del factor de comportamiento sísmico de 1 a 4 . Estos últimos se tomarán como referencia de la capacidad dúctil de estructuras que establece el RCDF-2004. Todas las estructuras se diseñaron para satisfacer el criterio de diseño columna fuerte-viga débil, aplicando el procedimiento de diseño por capacidad para columnas estipulado en el RCDF-2004. 
Se utilizó el modelo de amortiguamiento de Rayleigh para modelar el amortiguamiento viscoso de las estructuras .Por tratarse de estructuras de concreto reforzado, se juzgó apropiado consideraren los análisis fracciones del $5 \%$ del amortiguamiento crítico, aplicadas en el primer modo y en un modo para el cual la masa participante acumulada excede el $90 \%$. Esto con la finalidad de que los modos superiores cuya contribución pueda ser potencialmente significativa, no estén sobre-amortiguados. Los valores de los parámetros lp y $\beta$ considerados en el modelo elástico simplificado de la estructura dañada son respectivamente, $h / 2$ y $2 \%$. En los análisis se ignoraron los efectos de la interacción suelo-estructura.

\section{Acciones sísmicas de diseño}

El procedimiento propuesto requiere del cálculo de espectros inelásticos de resistencia para los valores requeridos del parámetro $\alpha$.Debido a que los espectros inelásticos estipulados en los reglamentos de diseño sísmico comúnmente se obtienen considerando comportamiento elastoplástico, en el presente estudio se usan como acciones sísmicas de diseño registros acelerográficos obtenidos en algunos sitios en particular, y sus correspondientes espectros elásticos e inelásticos de respuesta

Se consideran dos tipos de acciones sísmicas, registros acelerográficos característicos de suelo blando y de suelo firme. Para suelo blando la demanda sísmica considerada como representativa de los movimientos sísmicos asociados al ELPC de estructuras ubicadas en el Valle de México, es la componente este-oeste del registro acelerográfico obtenido en la estación SCT durante el sismo del 19 de septiembre de 1985. Este registro se muestra en la fig. 8a, y sus espectros de respuesta elásticos de seudoaceleración $(\mathrm{Sa})$ y desplazamiento $(S d)$ se muestran en la fig. 8 b. La demanda sísmica representativa de los movimientos sísmicos asociados al ELS, es la componente este-oeste del registro acelerográfico obtenido en la misma estación durante el sismo del 25 de abril de 1989. Este registro se muestra en la fig. $8 \mathrm{c}$, y sus espectros de respuesta elásticos de $S a$ y $S d$, se muestran en la fig. 8d. Todos los espectros de respuesta se calcularon para una fracción del amortiguamiento crítico del $5 \%$.

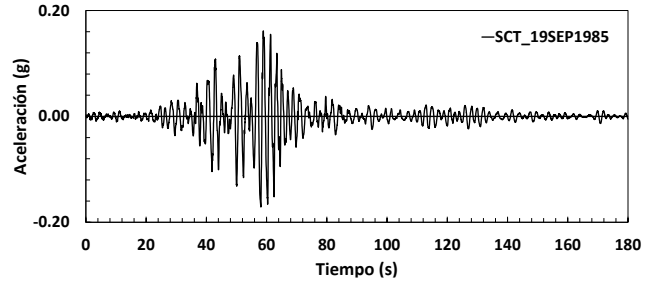

a). Registro acelerográfico en la estación SCT del sismo del 19 de septiembre de 1985

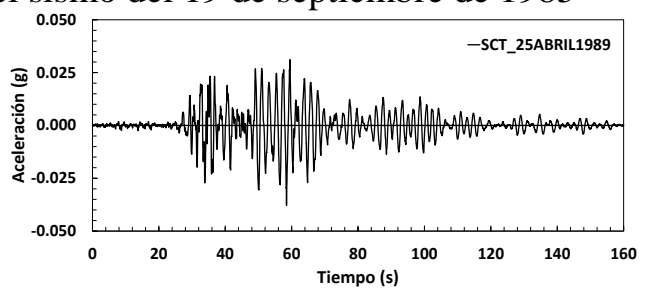

c). Registro acelerográfico en la estación SCT del sismo del 25 de abril de 1989

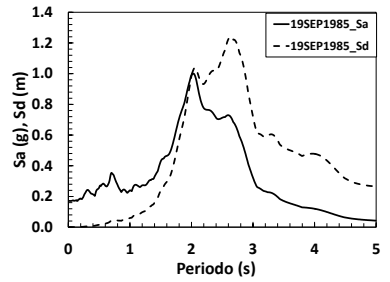

b). Espectros de respuesta elásticos

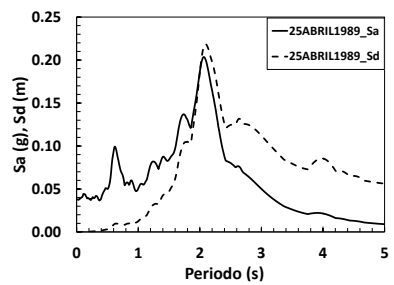

d). Espectros de respuesta elásticos

Figura 8. Registros acelerográficos y correspondientes espectros de respuesta elásticos de los niveles de diseño sísmico considerados en la estación SCT del Valle de México

Como demanda sísmica característica de suelo firme se considera la componente norte-sur del registro acelerográfico obtenido en El Centro, California, durante el sismo de Imperial Valley, del 18 de Mayo de 1940.Lasdemandas sísmicas de diseño asociadas a los ELPC y ELS se obtienen escalando el 
registro de El Centro, de tal forma que la máxima ordenada de su espectro de respuesta elástico de seudoaceleración sea igual a las correspondientes ordenadas de los espectros de los registros de los sismos del 19 de septiembre de 1985 y del 25 de abril de 1989, respectivamente. Los registros acelerográficos así escalados y sus espectros de respuesta elásticos de Sa y Sd se muestran en la fig. 9.

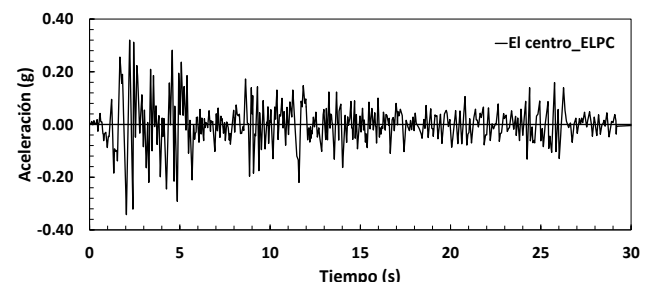

a). Registro acelerográfico usado para el ELPC

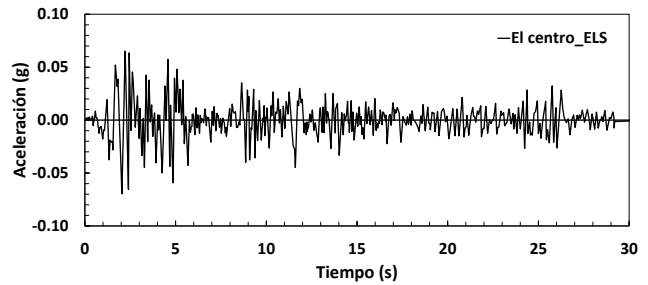

c). Registro acelerográfico usado para el ELS

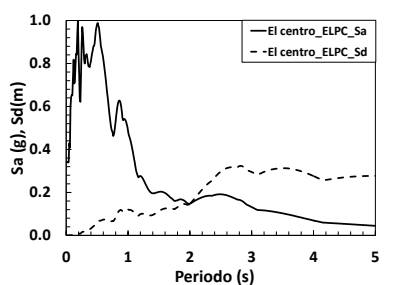

b). Espectros de respuesta

elásticos

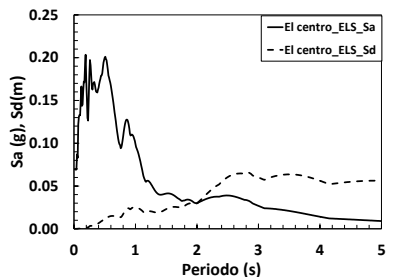

d). Espectros de respuesta elásticos

Figura 9. Registros acelerográficos escalados y correspondientes espectros de respuesta elásticos para los niveles de diseño sísmico considerados en el sitio El Centro.

\section{Descripción y discusión de los ejemplos de aplicación}

El marco de 17 niveles y el 12 niveles verticalmente irregular se diseñaron para el par de demandas sísmicas registradas en la estación SCT y se denominan ejemplos de aplicación 1 y 2 (marco E1 y marco E2). Los marcos de 12 niveles con el primer entrepiso "suave" y el de 8 niveles se diseñaron para el par de demandas sísmicas correspondientes al sitio de El Centro, y se denominan como los ejemplos de aplicación 3 y 4 (marco E3 y marco E4).

Para el diseño de estos marcos se siguió el procedimiento descrito anteriormente, haciendo las siguientes consideraciones adicionales:

Durante la aplicación del procedimiento se observó que en los marcos E3 y E4 la contribución de los modos superiores era significativa. Debido a esto, el dimensionamiento propuesto para las vigas y columnas se estableció de tal forma que la máxima distorsión de entrepiso en las estructuras correspondiente a su fluencia incipiente se presentara en sus entrepisos inferiores.

En los marcos E1, E2 y E4, se consideró una $\gamma_{E L S}=0.4 \%$, mientras que en el marco E3, por restricciones reglamentarias en las dimensiones mínimas de las secciones transversales de los elementos estructurales, se tuvo que considerar una $\gamma_{E L S}=0.2 \%$.

Aprovechando el hecho de que los sismos de diseño se definieron por medio de registros acelerográficos, las máximas ductilidades de desplazamiento de los modos superiores se obtuvieron como a continuación se describe. Se caracterizan los osciladores de 1 GDL que representan a los modos por medio de sus propiedades $T_{E}, R y / m$ y $\alpha$ previamente definidas, y se le realiza un análisis dinámico inelástico paso a paso considerando como excitación el acelerograma que define la demanda 
sísmica de diseño asociada al ELPC. La máxima ductilidad obtenida de dicho análisis es la ductilidad que desarrollará cada modo.

Los análisis modales espectrales se realizaron con el programa SAP 2000 (CSI, 2010).

En la tabla 2 se muestran las dimensiones requeridas para las secciones transversales de los elementos estructurales, y en la tabla 3algunas propiedades dinámicas y parámetros de diseño de los marcos estudiados. En estas tablas $T_{I S}$ es el periodo fundamental de las estructuras en el ELS. En la tabla 4 se muestran algunas propiedades dinámicas de los tres primeros modos de las estructuras diseñadas, así como algunas de sus propiedades globales. En la fig. 13 se muestran las distribuciones de daño propuestas para las estructuras estudiadas. En las figs. 10 y 16 se muestran, respectivamente, las curvas de comportamiento de los tres primeros modos de las estructuras diseñadas y sus curvas de capacidad.

Tabla 2. Dimensiones de las secciones transversales de los elementos estructurales de los marcos

\begin{tabular}{|c|c|c|c|c|c|c|c|c|}
\hline & \multicolumn{2}{|c|}{ Marco E1 } & \multicolumn{2}{|c|}{ Marco E2 } & \multicolumn{2}{|c|}{ Marco E3 } & \multicolumn{2}{|c|}{ Marco E4 } \\
\hline & Nivel & $\begin{array}{l}\text { Dimensiones } \\
(\mathrm{m})\end{array}$ & Nivel & $\begin{array}{l}\text { Dimensiones } \\
(\mathrm{m})\end{array}$ & Nivel & $\begin{array}{c}\text { Dimensiones } \\
\text { (m) }\end{array}$ & Nivel & $\begin{array}{c}\text { Dimensiones } \\
\text { (m) }\end{array}$ \\
\hline \multirow{4}{*}{ Columnas } & 1 a 7 & $1.10 \times 1.10$ & $1 \mathrm{a} 2$ & $0.80 \times 0.80$ & 1 a 6 & $0.70 \times 0.70$ & 1 a 8 & $0.45 \times 0.45$ \\
\hline & 8 a 11 & $0.90 \times 0.90$ & 3 a 8 & $0.70 \times 0.70$ & 7 a 12 & $0.50 \times 0.50$ & & \\
\hline & 12 a 14 & $0.75 \times 0.75$ & 9 a 12 & $0.60 \times 0.60$ & & & & \\
\hline & 15 a 17 & $0.60 \times 0.60$ & & & & & & \\
\hline \multirow{2}{*}{ Vigas } & 1 a 9 & $0.40 \times 0.85$ & 1 a 7 & $0.35 \times 0.60$ & 1 a 12 & $0.30 \times 0.55$ & 1 a 8 & $0.25 \times 0.45$ \\
\hline & 10 a 17 & $0.40 \times 0.75$ & 8 a 12 & $035 \times 0.45$ & & & & \\
\hline
\end{tabular}

Tabla 3. Algunas propiedades dinámicas y parámetros de diseño de los marcos

\begin{tabular}{cllllllllll}
\hline Marco & $\begin{array}{l}\mathrm{T}_{1 \mathrm{~S}} \\
(\mathrm{~s})\end{array}$ & $\begin{array}{l}\gamma_{\mathrm{y}} \\
(\%)\end{array}$ & $\begin{array}{l}\mathrm{T}_{1 \mathrm{E}} \\
(\mathrm{s})\end{array}$ & $\begin{array}{l}\mathrm{R}_{\mathrm{y}} / \mathrm{m}_{1} \\
\left(\mathrm{~m} / \mathrm{s}^{2}\right)\end{array}$ & $\begin{array}{l}\mathrm{Sd}_{\mathrm{y} 1} \\
(\mathrm{~m})\end{array}$ & $\begin{array}{l}\gamma_{\mathrm{ELPC} 1} \\
(\%)\end{array}$ & $\begin{array}{l}\alpha_{1} \\
(\%)\end{array}$ & $\mu_{1}$ & $\begin{array}{l}\mathrm{T}_{1 \mathrm{D}} \\
(\mathrm{s})\end{array}$ & $\begin{array}{l}\gamma_{\mathrm{ELPC}} \\
(\%)\end{array}$ \\
\hline $\mathrm{E} 1$ & 1.90 & 0.67 & 2.24 & 1.704 & 0.217 & 1.5 & 4.72 & 2.38 & 10.3 & 1.54 \\
$\mathrm{E} 2$ & 1.72 & 0.84 & 2.06 & 1.943 & 0.208 & 1.8 & 5.10 & 2.32 & 9.12 & 1.90 \\
$\mathrm{E} 3$ & 2.30 & 0.69 & 2.67 & 0.903 & 0.163 & 1.1 & 5.90 & 1.69 & 10.99 & 1.13 \\
$\mathrm{E} 4$ & 2.31 & 0.98 & 2.55 & 0.797 & 0.131 & 1.7 & 9.63 & 1.79 & 8.22 & 1.67 \\
\hline
\end{tabular}

Tabla 4. Propiedades y parámetros de respuesta modales y globales de los marcos

\begin{tabular}{|c|c|c|c|c|c|c|c|c|c|c|c|}
\hline Marco & Modo & $\mathrm{T}_{\mathrm{E}}$ & $\mathrm{T}_{\mathrm{D}}$ & $\begin{array}{l}\mathrm{Ry} / \mathrm{m} \\
\left(\mathrm{m} / \mathrm{s}^{2}\right)\end{array}$ & $\alpha(\%)$ & $\mu$ & $\begin{array}{c}\text { Rcomp } / \mathrm{m} \\
\left(\mathrm{m} / \mathrm{s}^{2}\right)\end{array}$ & $\Gamma_{D} / \Gamma_{E}$ & $\frac{\text { Vbmax }}{\text { Vby }}$ & $\begin{array}{c}\alpha \text { global } \\
(\%)\end{array}$ & $\mu$ global \\
\hline \multirow{3}{*}{ E1 } & 1 & 2.24 & 10.3 & 1.70 & 4.72 & 2.38 & 0.110 & 1.02 & \multirow{3}{*}{1.07} & \multirow{3}{*}{5.05} & \multirow{3}{*}{2.45} \\
\hline & 2 & 0.79 & 2.36 & 1.50 & 11.3 & 2.13 & 0.192 & 0.98 & & & \\
\hline & 3 & 0.43 & 0.87 & 1.50 & 19.8 & 1.83 & 0.247 & 1.03 & & & \\
\hline \multirow{3}{*}{ E2 } & 1 & 2.05 & 9.12 & 1.94 & 5.10 & 2.32 & 0.130 & 1.04 & \multirow{3}{*}{1.08} & \multirow{3}{*}{6.51} & \multirow{3}{*}{2.55} \\
\hline & 2 & 0.78 & 2.20 & 1.52 & 12.7 & 2.02 & 0.197 & 1.01 & & & \\
\hline & 3 & 0.42 & 0.85 & 1.48 & 24.8 & 1.72 & 0.265 & 0.84 & & & \\
\hline \multirow{3}{*}{ E3 } & 1 & 2.67 & 10.9 & 0.90 & 5.90 & 1.69 & 0.036 & 1.02 & \multirow{3}{*}{1.08} & \multirow{3}{*}{10.0} & \multirow{3}{*}{1.66} \\
\hline & 2 & 0.88 & 2.06 & 2.91 & 18.4 & 1.53 & 0.283 & 0.93 & & & \\
\hline & 3 & 0.48 & 0.85 & 4.12 & 32.2 & 1.69 & 0.909 & 1.00 & & & \\
\hline \multirow{3}{*}{ E4 } & 1 & 2.55 & 8.22 & 0.79 & 9.60 & 1.79 & 0.060 & 1.04 & \multirow{3}{*}{1.11} & \multirow{3}{*}{15.5} & \multirow{3}{*}{1.75} \\
\hline & 2 & 0.78 & 1.45 & 2.25 & 28.9 & 1.74 & 0.483 & 0.89 & & & \\
\hline & 3 & 0.41 & 0.57 & 4.75 & 51.4 & 1.53 & 1.286 & 0.74 & & & \\
\hline
\end{tabular}




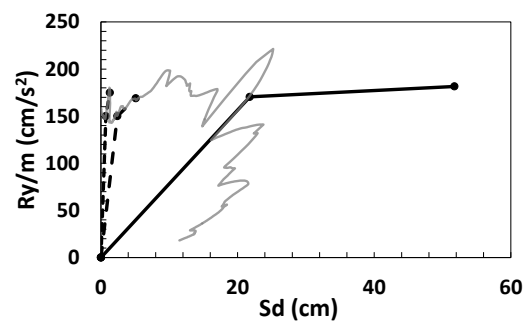

a) Marco E1

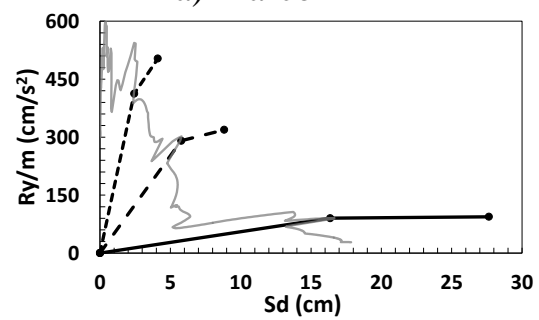

c) Marco E3

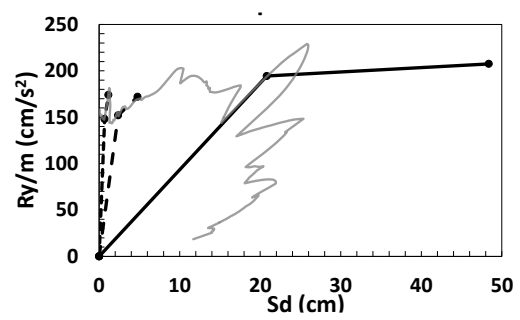

b) Marco E2

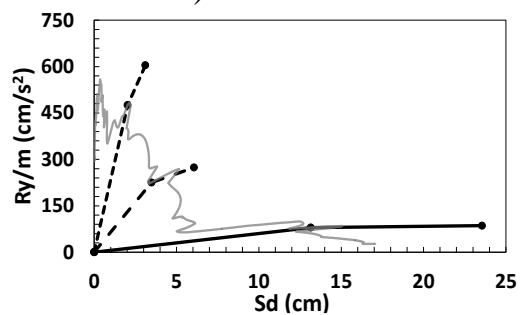

d) Marco E4

Figura 10. Curvas de comportamiento de los tres primeros modos de los marcos

En la figs. 10a y 10b se observa que los desplazamientos de fluencia de los modos superiores de los marcos E1 y E2 son considerablemente menores que los de sus modos fundamentales. Esto se debe a que la rama ascendente del espectro de desplazamiento del acelerograma registrado en la estación SCT, en la que se ubican los periodos elásticos de todos los modos de ambas estructuras, presenta una pendiente pronunciada. Como consecuencia, la contribución de los modos superiores al valor total de las distorsiones de entrepiso en el EFI es mínima, figs. 11a y 11b. Por el contrario, en la figs. 10c y 10d se observa que los desplazamientos de fluencia de los modos superiores de los marcos E3 y E4 son fracciones significativas del desplazamiento correspondiente a su modo fundamental. Esto se debe a que la rama ascendente del espectro de desplazamiento del acelerograma registrado en el sitio El centro, en la que también se ubican los periodos elásticos de todos los modos de ambas estructuras, presenta una pendiente poco pronunciada. Como resultado, la contribución de los modos superiores al valor total de las distorsiones de entrepiso en el EFI es considerable, figs. 11c y 11d.

La comparación de la magnitud en que contribuyen los modos superiores a las distorsiones de entrepiso de, por ejemplo, los marcosE1 y E4 indican que dicha contribución depende esencialmente de la magnitud relativa de las ordenadas de desplazamiento correspondientes a los distintos modos, más que de la altura de la estructura. En la tabla 4 se observa que las ductilidades desarrolladas por los modos superiores son menores a la del modo fundamental, y que los factores de participación modales de las estructuras en su estado dañado son ligeramente menores a los que presenta en su estado elástico. Debido a esto, la contribución de los modos superiores a las distorsiones de entrepiso en el ELPC son moderadamente menores que en el EFI. 


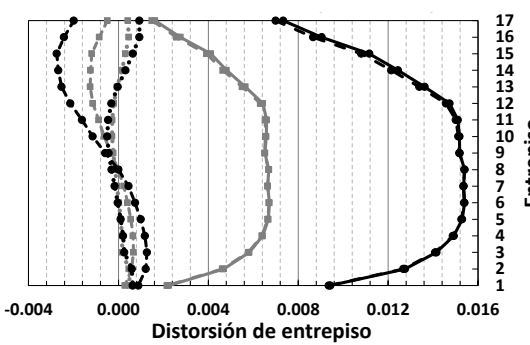

a) Marco E1

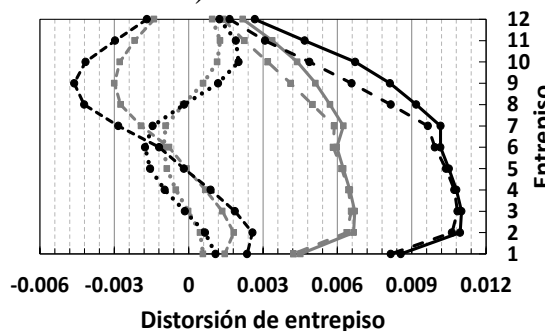

c) Marco E3

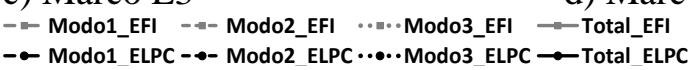

Figura 10. Distorsiones de entrepiso modales de diseño correspondientes a los estados límite de fluencia incipiente y de prevención del colapso

\section{VALIDACIÓN ANÁLITICA DEL PROCEDIMIENTO}

Con la finalidad de demostrar con mayor claridad la eficacia y validez del procedimiento propuesto considerando la resistencia de fluencia de todos los modos y por tanto, su contribución, con la mayor similitud posible a como se establecieron en el diseño, en este estudio se adopta el siguiente criterio recomendado en algunos códigos para verificar el diseño sísmico de una estructura.

Dicho criterio consiste en compararlos parámetros de respuesta de una estructura diseñada para cada estado límite, con el promedio de, o, sus máximos valores obtenidos de análisis dinámicos inelásticos paso a paso (ADIPP) de esta estructura sujeta a un conjunto de acelerogramas. El promedio delas ordenadas de los espectros de respuesta elásticos de seudoaceleración de estos registros iguala, en cierto intervalo de periodos, las ordenadas de los correspondientes espectros que definieron para cada estado límite las acciones sísmicas de diseño. El intervalo de periodos considerado comprende desde el periodo elástico del modo superior que se considere tiene una contribución aún significativa, hasta el periodo fundamental elástico de la estructura.

De acuerdo a la recomendación del Código Uniforme de Construcciones de 1994 (UBC, 1994), si el conjunto comprende al menos siete registros acelerográficos, los parámetros de respuesta dela estructura diseñada se deben comparar con el promedio de los máximos valores obtenidos de los ADIPP, los cuales se consideran como los valores exactos.

\section{Registros acelerográficos considerados}

Con la finalidad de igualar el escenario sísmico de diseño, los acelerogramas usados para obtener los ajustados fueron registrados en la misma estación, y corresponden a sismos de magnitudes similares provenientes de la misma fuente del sismo cuyo acelerograma se usó como sismo de diseño para el estado límite considerado. De acuerdo a los valores de los periodos $\mathrm{T}_{\mathrm{E}}$ de las estructuras diseñadas mostrados en la tabla 4 , se consideró apropiado un intervalo de periodos de 0.20 a $4 \mathrm{~s}$ para obtener los acelerogramas 
ajustados. Seis de los acelerogramas que contiene el conjunto, son señales reales ajustadas con el método de las ondoletas propuesto por Abrahamson (1992) y Hancock et al. (2006) e implementado en el programa SeismoMatch (Seismosoft, 2012). El séptimo acelerograma del conjunto es el que se usó como acción sísmica de diseño para el estado límite correspondiente. En la fig. 12 se muestra que el promedio de los espectros de respuesta elásticos se seudoaceleración de estos registros, calculados para una fracción del 5\% del amortiguamiento crítico, es aproximadamente igual al espectro del registro usado como acción sísmica de diseño para cada estado límite considerado.

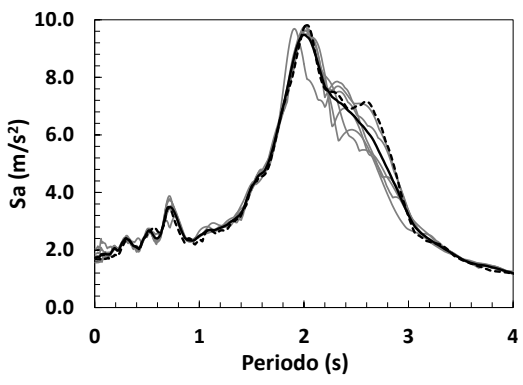

a). Estación SCT (ELPC)

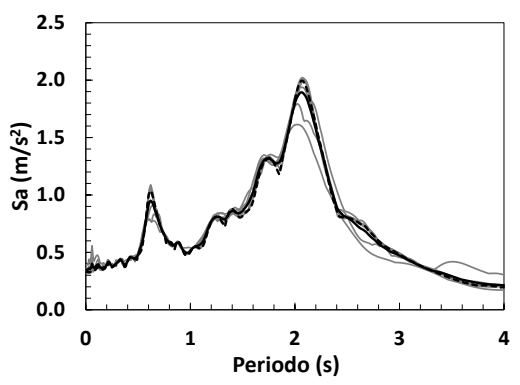

b). Estación SCT (ELS)

-Espectros de los acelerogramas ajustados ---Espectro del acelerograma de diseño -Promedio de los espectros

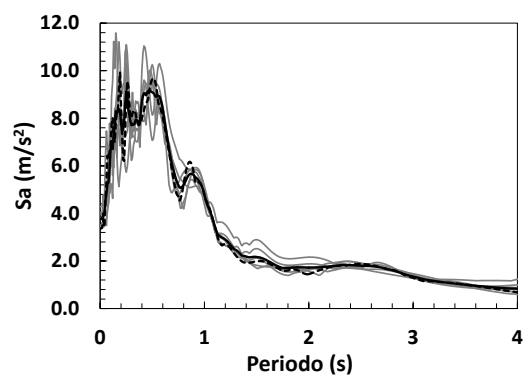

c). Estación El centro (ELPC)

Figura 12. Espectros de respuesta elásticos de seudoaceleración de los registros acelerográficos ajustados y usados para validar el procedimiento

\section{Modelos matemáticos de las estructuras para los análisis dinámicos inelásticos paso a paso}

En los modelos matemáticos de las estructuras sobre los que se realizan los análisis inelásticos se utilizan los mismos valores de los parámetros $E I_{\text {eff, }} l p$ y $\beta$ considerados en el diseño. Además se consideró que las articulaciones plásticas presentan un comportamiento histerético bilineal sin degradación de rigidez ni resistencia, y las resistencias asignadas a las vigas y a las columnas de la base son exactamente iguales a las obtenidas del análisis. La razón de estos criterios se debe a que si consideran los valores más aproximados de dichas propiedades obtenidos de los diagramas momento-curvatura de las secciones diseñadas, se modificarían los periodos y los cortantes basales resistentes de las estructuras obtenidos del análisis, y consecuentemente los valores de todos los parámetros de diseño. Las resistencias asignadas a las columnas de todos los niveles a excepción de las del primero, son las obtenidas al diseñarlas por capacidad. Se utilizó el modelo de Rayleigh proporcional a las matrices de masa y rigidez inicial para modelar el amortiguamiento de las estructuras. Las fracciones de amortiguamiento y los modos en los que éstas se aplican, son las mismas que se consideraron en el diseño. Los análisis no lineales paso a paso se realizaron con el programa de análisis inelástico de estructuras bidimensionales Ruaumoko-2D (Carr, 2000).

\section{Presentación y examen de resultados}

Los errores relativos en los parámetros de desempeño cuantificables se calculan mediante la ec. 14:

$$
E R(\%)=\left[\left(P D \_ \text {diseno }-P D \_ \text {demandado }\right) / P D_{-} \text {demandado }\right] 100
$$

donde: $P D \_$diseño es el parámetro de desempeño de la estructura obtenido del diseño para un estado límite, y $P D \_d e m a n d a d o$ es el promedio de los máximos parámetros de desempeño, obtenidos de los 
ADIPP de la estructura sujeta al conjunto de acelerogramas representativos de la demanda sísmica de diseño correspondiente a cada estado límite.

\section{Distribuciones de daño}

En la fig. 13 se compara la distribución de articulaciones plásticas propuesta en cada marco, con la representativa de las desarrolladas en el ELPC. Se observa que en todas las estructuras se desarrollaron las articulaciones plásticas propuestas en las vigas. Al diseñar por capacidad las columnas delos marcos E1, E2 y E3 se logra que solo se desarrollen articulaciones plásticas en las columnas de la base. En algunas columnas de los niveles superiores del marco E4, sin embargo, se presenta una limitada fluencia.

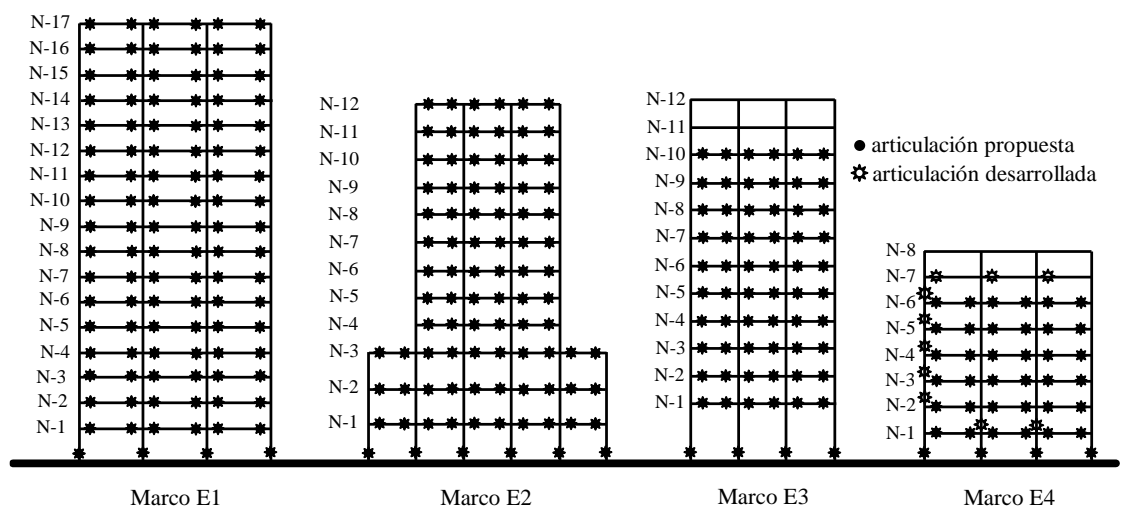

Figura 13. Comparación de la distribución de articulaciones plásticas propuesta para cada marco con la representativa de las desarrolladas ante el sismo de diseño asociado al ELPC

\section{Desplazamientos laterales de los niveles}

El desplazamiento lateral máximo de los niveles de un edificio es el parámetro de desempeño usado para evitar el impacto entre edificios adyacentes, y aunque no es el parámetro de referencia para el diseño, se muestra la precisión con la que el procedimiento propuesto puede estimar sus valores. En la fig. 14 se comparan los desplazamientos de diseño de los niveles para el ELPC, con los correspondientes máximos demandados, los cuales generalmente ocurren en diferentes tiempos. Se observa que los desplazamientos de los marcos E1, E2 y E3 se sobrestiman ligeramente, mientras que los del marco E4 se subestiman. Estos resultados son consistentes con el hecho de que en los marcos E1, E2 y E3 se desarrollaron las distribuciones de daño propuestas, por lo que los desplazamientos de diseño definen un límite superior razonable de las máximas demandas de deformación lateral. Mientras que en el marco E4 se desarrollaron un mayor número de articulaciones plásticas que las propuestas, conduciendo a que los desplazamientos demandados fueran mayores a los de diseño. Los errores relativos en los desplazamientos de la azotea varían de $-15 \%$ a $13 \%$.

\section{Distorsiones de entrepiso}

En la fig. 15 se comparan las distorsiones de entrepiso de diseño para ambos estados límite, con las correspondientes máximas demandadas, las cuales generalmente ocurren en diferentes tiempos. En la misma figura también se muestran la $\gamma_{y}$ de cada uno de los entrepisos. De esta figura pueden hacerse las siguientes observaciones: (1), tanto el perfil de distorsiones como la máxima distorsión demandada en el ELS son razonablemente similares a los estimados en el diseño. (2), las tendencias que se presentan en el perfil de distorsiones de entrepiso en el ELPC son, en general, consistentes con las del perfil de desplazamientos laterales de los niveles, es decir, las distorsiones de entrepiso de los marcos E1, E2 y E3 
se sobrestiman ligeramente, mientras que las del marco E4 se subestiman moderadamente. El error relativo en la máxima distorsión de diseño de los marcos E1, E2 y E3 en el ELPC, se encuentran entre $\pm 8 \%$, mientras que en el marco E4 es de-22\%. (3), en el marco E3 las distorsiones demandadas en los entrepisos superiores, en los cuales la contribución de los modos superiores es más significativa, son mayores a las diseño. Además, las mayores deferencias entre las distorsiones demandadas y las de diseño en el marco E4 se presentan en los entrepisos superiores. Estos hechos indican que mediante la aplicación del procedimiento propuesto se subestiman moderadamente las demandas de deformación en los entrepisos en los que la contribución de los modos superiores es significativa. (4), en la fig. 15a la máxima distorsión demandada en el entrepiso superior del marco E1 es menor que su $\gamma_{y}$, lo que indica que las vigas de dicho entrepiso no fluirán.

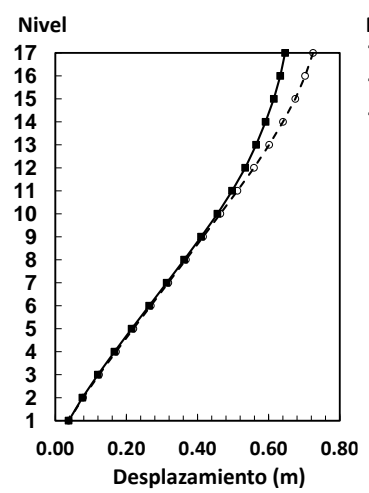

a) Marco E1

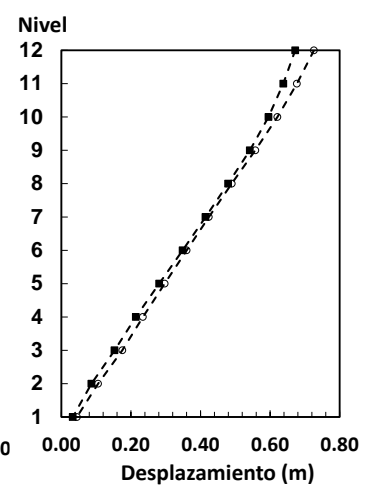

b) Marco E2

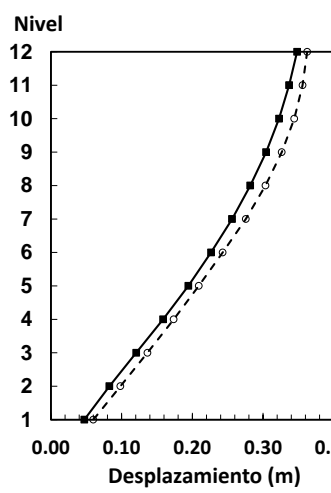

c) Marco E3

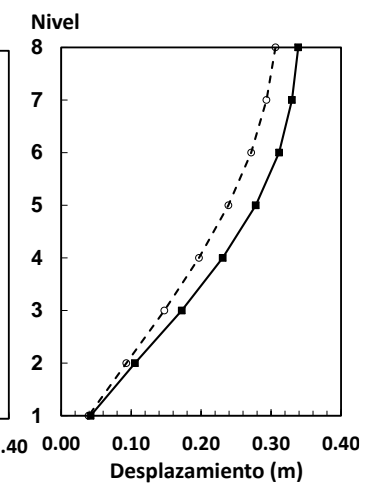

d) Marco E4

Figura 14. Comparación de los desplazamientos laterales de diseño de los niveles para ELPC, con el promedio de los máximos demandados ante la correspondiente acción sísmica

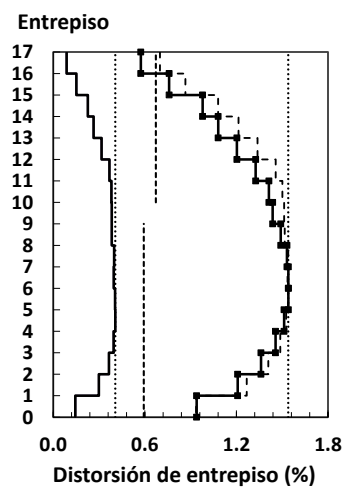

a) MarcoE1

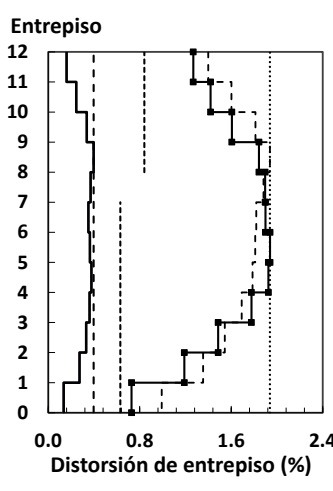

b) Marco E2

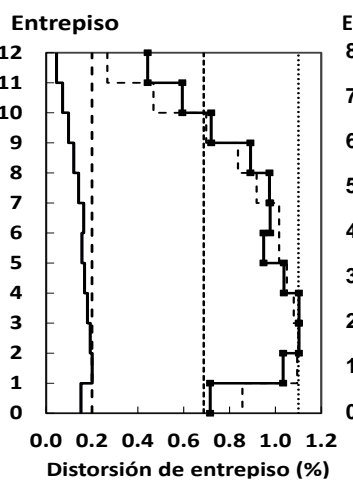

c) Marco E3

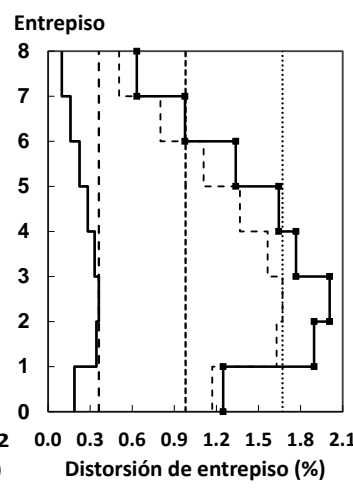

c) Marco E4

—Diseño ELS - - Diseño ELPC - ADIPP_bilineal - - ADIPP_Takeda - - Permisible ELS ---Fluencia incipiente -..... Permisible ELPC

Figura 15. Comparación de las distorsiones de diseño para ambos estados límite con el promedio de las máximas demandas ante sus correspondientes acciones sísmicas

\section{Curvas de capacidad}

En la fig. 16 se comparan las curvas de capacidad de diseño de los cuatro marcos, con sus respuestas promedio en el tiempo cortante basal-desplazamiento lateral de la azotea. Se puede observar que el cortante basal de diseño y el desplazamiento máximo de la azotea asociado representan razonablemente bien la envolvente en el tiempo de los correspondientes parámetros de respuesta. Los 
errores relativos de los máximos cortantes basales de diseño de los marcos E1, E2, E3 y E4 son, respectivamente: $1.88 \%,-11.38 \%,-13.47 \%$ y $-20.32 \%$, los cuales se pueden considerar aceptables. En la décima columna de la tabla 4se muestran los valores de los cocientes Vbmax/Vby, los cuales indican que de no considerarse el cortante basal complementario dado por la rigidez de posfluencia de la estructura, los errores en la estimación de Vbmax pueden ser inaceptables.

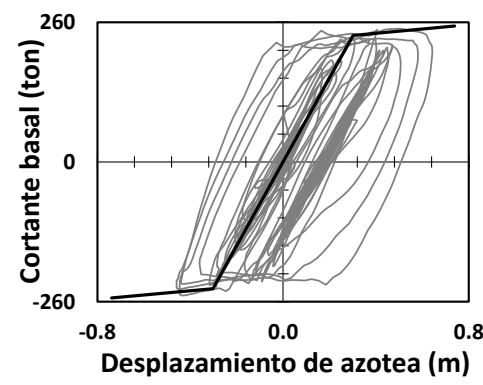

a) Marco E1

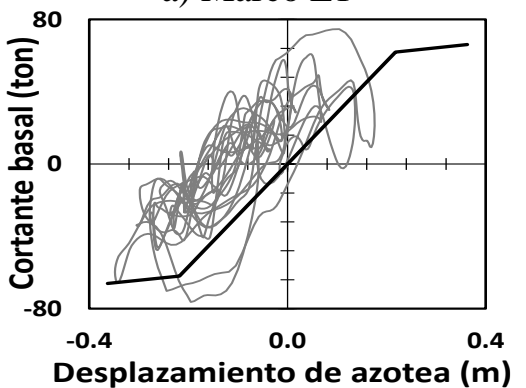

c) Marco E3

- Curva de capacidad de diseño

- Respuesta en el tiempo Cortante basal vs Desplazamiento de la azotea_bilineal

- Respuesta en el tiempo cortante basal vs desplazamiento de la azotea_Takeda

Figura 16. Curvas de capacidad de diseño y respuestas promedio en el tiempo: cortante basal- desplazamiento de azotea.

\section{CONCLUSIONES Y RECOMENDACIONES}

En este trabajo se presentó e ilustró la aplicación de un procedimiento de diseño sísmico multinivel basado en desplazamientos para estructuras formadas por marcos de concreto reforzado, que permite considerar explícitamente tres estados límite y sus correspondientes niveles de diseño sísmico. Con el propósito de validar los resultados obtenidos, se efectuaron ADIPP delas estructuras diseñadas considerando un conjunto de acelerogramas cuyos espectros de respuesta elásticos de seudoaceleración son compatibles con los espectros usados como acciones sísmicas de diseño para cada estado límite. Del análisis de los resultados se puede concluir lo siguiente:

En los dos estados límite de diseño el método propuesto estimó con aceptable aproximación, tanto el perfil de distorsiones como la máxima distorsión de entrepiso demandadas en las estructuras estudiadas, lo que muestra su eficacia para predecir las demandas de deformación lateral. En cuanto a fuerzas de diseño, el procedimiento estimó con razonable precisión los máximos cortantes basales de las estructuras estudiadas.

De acuerdo con los resultados anteriores, se puede concluir que el criterio empleado para definir las resistencias de los modos superiores es apropiado. 
También, para fines de diseño, se puede considerar razonable suponer que todas las articulaciones plásticas requeridas se desarrollan de manera simultánea, incluso en estructuras en las que la contribución de los modos superiores es significativa.

Es necesario adecuar el procedimiento propuesto para extender su aplicación a estructuras tridimensionales en las que se deban de considerar los efectos de interacción suelo-estructura, así como también extender su aplicación a estructuras conformadas por marcos y muros, y muros de concreto reforzado.

\section{AGRADECIMIENTOS}

Se agradece al Consejo Nacional de Ciencia y Tecnología las becas otorgadas al primer autor de este artículo para la realización de sus estudios de posgrado y el patrocinio del proyecto CONACyT \# 82839 "Desarrollo del marco conceptual, modelos teóricos y métodos simplificados para la evaluación y el diseño sísmico de estructuras basado en desempeño" en el que se basa este trabajo.

\section{REFERENCIAS}

Abrahamson, N A (1992), "Non-stationary spectral matching",Seismological Research Letters, Vol. 63, No. 1, pp. 30-46.

Barradas, J E (2014), "Procedimiento de diseño sismico multinivel basado en desplazamiento para estructuras a base de marcos de concreto reforzado", Tesis de Doctorado, Programa de Maestría y Doctorado en Ingeniería, UNAM.

Bertero, R (2001), “Diseño Sismorresistente basado en la Performance”,Tesis de Doctorado, Universidad de Buenos Aires, Argentina.

Carr, A J (2000), "Ruaumoko, Inelastic Dynamic Analysis Computer Program and Users Manual", University of Canterbury, Department of Civil Engineering, Nueva Zelanda.

Chopra, A K y R K Goel (2001), “A Modal Pushover Analysis Procedure to Estimate Seismic Demands for Buildings: Theory and Preliminar Evaluation", Pacific Earthquake Engineering Research Center, PEER 2001/03.

Chopra, A.K. (2005), Dynamics of structures, Theory and applications to earthquake engineering, Editorial Prentice Hall, Segunda Edición.

CSI (2010),"SAP2000, Integrated finite element analysis and design of structures", Computers and Structures, Inc., Berkeley, California.

FIB (2003), "Displacement-based seismic design of reinforced concrete buildings", Federación internacional para concreto estructural, Boletin 25, Grupo de trabajo 7.2.

García, L E, A Perez y J Bonacci (1996), "Cost implications of drift controlled design of reinforced concrete buildings", Memorias $11^{\text {th }}$ World Conference on Earthquake Engineering, México.

Hancock, J, J Watson-Lamprey, N A Abrahamson, J J Bommer, A Markatis, E McCoy y R Mendis (2006), "An improved method of matching response spectra of recorded earthquake ground motion using wavelets", Journal of Earthquake Engineering, Vol. 10, pp. 67-89.

Kappos, A J y A Manafpour (2001),'Seismic Design of R/C buildings with the aid of advanced analytical 
techniques”, Engineering Structures, Vol. 23, pp. 319-332.

Mayes, R L (1995), “Interstorydrift design and damage control issues”, The Structural Design of Tall buildings, Vol.4, pp. 15-25.

Moehle, J P (1992), "Displacement- Based Design of RC Structures Subjected to Earthquakes", Earthquake Spectra, Vol. 8, No. 3, pp. 403-428.

Otani, S (2002), "Nonlinear Earthquake Response Analysis of Reinforced Concrete Buildings", Apuntes para la European School for Advanced Studies in Reduction of Seismic Risk, Instituto Universitario, di Studi Superiori di Pavia, Universita de gli Studi di Pavia, Pavia, Italia.

Park, R y T Paulay (1975), Reinforced Concrete Structures, John Wiley \& Sons Inc., New York.

Priestley, M J N (1998), "Brief comments on elastic flexibility of reinforced concrete frames and significance to seismic design", Bulletin of the New Zealand National Society for Earthquake Engineering,Vol. 3, No. 4, pp. 426-259.

Priestley, M J N, G M Calvi, y M J Kowalsky (2007), Displacement-Based Seismic Design of Structures, Instituto Universitario de Estudios Superiores de Pavia, Italia.

Qi, X y J P Moehle (1991), "Displacement Design Approach for Reinforced Concrete Structures Subjected to Earthquakes", Report No. UCB/EERC-91/02, Earthquake Engineering Research Center, Universidad de California en Berkeley.

RCDF (2004), "Reglamento de Construcciónes para el Distrito Federal, Cuerpo principal y sus Normas Técnicas Complementarias", Gaceta oficial del departamento del Distrito Federal, México.

Rodriguez, M E, J I Restrepo y A J Carr (2002), "Earthquake-induced floor horizontal accelerations in buildings", Earthquake Engineering and Structural Dynamics, Vol. 31, pp. 693-718.

Ruiz-García, J y E Miranda (2003), "Inelastic displacement ratios for evaluation of existing structures", Earthquake Engineering and Structural Dynamics, Vol. 32, pp.1237-1258.

Ruiz-García, J y E Miranda (2006), “Inelastic displacement ratios for evaluation of structures built on soft soil sites”, Earthquake Engineering and Structural Dynamics, Vol. 35, pp. 679-694.

SeismoSoft (2012), "SeismoMatch, A computer program for adjusting earthquake accelerograms to match a specific target response spectrum", Available from URL:http://www.seismosoft.com.

Sullivan, T J, M J N Priestley y G M Calvi (2008), "Estimating the Higher-Mode Response of Ductile Structures", Journal of Earthquake Engineering, Vol.12, pp. 456-472.

Uang, C (1991), "Establishing R (or Rw) and Cd Factors for Buildings Seismic Provisions", Journal of Structural Engineering, Vol. 117, No.1, pp. 19-28.

UBC (1994), “Uniform Building Code1994", International Conference of Buildings Officials, Washington, D.C. 